\title{
A search for ultra-high energy neutrinos in highly inclined events at the Pierre Auger Observatory
}

P. Abreu, ${ }^{1}$ M. Aglietta,${ }^{2}$ M. Ahlers,${ }^{3}$ E.J. Ahn,${ }^{4}$ I.F.M. Albuquerque, ${ }^{5}$ D. Allard,${ }^{6}$ I. Allekotte,${ }^{7}$ J. Allen, ${ }^{8}$ P. Allison, ${ }^{9}$ A. Almela,${ }^{10,11} \mathrm{~J}$. Alvarez Castillo, ${ }^{12} \mathrm{~J}$. Alvarez-Muñiz ${ }^{13} \mathrm{M}$. Ambrosio, ${ }^{14}$ A. Aminaei,${ }^{15}$

L. Anchordoqui ${ }^{16}$ S. Andringa, ${ }^{1}$ T. Anticic,${ }^{17}$ C. Aramo, ${ }^{14}$ E. Arganda,${ }^{18,19}$ F. Arqueros,${ }^{19}$ H. Asorey, ${ }^{7}$ P. Assis,${ }^{1}$ J. Aublin, ${ }^{20}$ M. Ave,${ }^{21}$ M. Avenier ${ }^{22}$ G. Avila, ${ }^{23}$ T. Bäcker, ${ }^{24}$ A.M. Badescu, ${ }^{25}$ M. Balzer, ${ }^{26}$ K.B. Barber,${ }^{27}$ A.F. Barbosa, ${ }^{28}$ R. Bardenet, ${ }^{29}$ S.L.C. Barroso, ${ }^{30}$ B. Baughman, ${ }^{9}$, 国 J. Bäuml, ${ }^{31}$ J.J. Beatty, ${ }^{9}$ B.R. Becker, ${ }^{32}$ K.H. Becker, ${ }^{33}$ A. Bellétoile,,${ }^{34}$ J.A. Bellido, ${ }^{27}$ S. BenZvi, ${ }^{3}$ C. Berat, ${ }^{22}$ X. Bertou, ${ }^{7}$ P.L. Biermann,${ }^{35}$ P. Billoir, ${ }^{20}$ F. Blanco, ${ }^{19}$ M. Blanco, ${ }^{36}$ C. Bleve, ${ }^{33}$ H. Blümer, ${ }^{21,31} \mathrm{M}$. Bohácová, ${ }^{37}$ D. Boncioli, ${ }^{38}$ C. Bonifazi,,${ }^{39,} 20$ R. Bonino, ${ }^{2}$ N. Borodai,${ }^{40}$ J. Brack, ${ }^{41}$ I. Brancus, ${ }^{42}$ P. Brogueira, ${ }^{1}$ W.C. Brown,${ }^{43}$ R. Bruijn, ${ }^{44}$, b P. Buchholz,${ }^{24}$ A. Bueno, ${ }^{45}$ R.E. Burton, ${ }^{46}$ K.S. Caballero-Mora ${ }^{47}$ B. Caccianiga ${ }^{48}$ L. Caramete, ${ }^{35}$ R. Caruso, ${ }^{49}$ A. Castellina, ${ }^{2}$ O. Catalano, ${ }^{50}$ G. Cataldi, ${ }^{51}$ L. Cazon, ${ }^{1}$ R. Cester,${ }^{52}$ J. Chauvin, ${ }^{22}$ S.H. Cheng, ${ }^{47}$ A. Chiavassa, ${ }^{2}$ J.A. Chinellato, ${ }^{53}$ J. Chirinos Diaz ${ }^{54}$ J. Chudoba ${ }^{37}$ R.W. Clay,${ }^{27}$ M.R. Coluccia, ${ }^{51}$ R. Conceição, ${ }^{1}$ F. Contreras, ${ }^{55}$ H. Cook, ${ }^{44}$ M.J. Cooper, ${ }^{27}$ J. Coppens, ${ }^{15,56}$ A. Cordier, ${ }^{29}$ S. Coutu, ${ }^{47}$ C.E. Covault,${ }^{46}$ A. Creusot,${ }^{6}$ A. Criss, ${ }^{47}$ J. Cronin, ${ }^{57}$ A. Curutiu, ${ }^{35}$ S. Dagoret-Campagne, ${ }^{29}$ R. Dallier,${ }^{34}$ S. Dasso, ${ }^{58,59}$ K. Daumiller,${ }^{31}$ B.R. Dawson, ${ }^{27}$ R.M. de Almeida, ${ }^{60}$ M. De

Domenico, ${ }^{49}$ C. De Donato, ${ }^{12}$ S.J. de Jong, ${ }^{15,56}$ G. De La Vega,${ }^{61}$ W.J.M. de Mello Junior, ${ }^{53}$ J.R.T. de Mello Neto, ${ }^{39}$ I. De Mitri, ${ }^{51}$ V. de Souza, ${ }^{62}$ K.D. de Vries,${ }^{63}$ L. del Peral,${ }^{36}$ M. del Río, ${ }^{38,55}$ O. Deligny, ${ }^{64}$ H. Dembinski, ${ }^{21}$ N. Dhital,${ }^{54}$ C. Di Giulio, ${ }^{65}$ M.L. Díaz Castro, ${ }^{66}$ P.N. Diep,${ }^{67}$ F. Diogo,${ }^{1}$ C. Dobrigkeit,${ }^{53}$ W. Docters, ${ }^{63}$ J.C. D'Olivo, ${ }^{12}$ P.N. Dong, ${ }^{67,64}$ A. Dorofeev ${ }^{41}$ J.C. dos Anjos, ${ }^{28}$ M.T. Dova, ${ }^{18}$ D. D'Urso, ${ }^{14}$ I. Dutan, ${ }^{35}$ J. Ebr, ${ }^{37}$ R. Engel, ${ }^{31}$ M. Erdmann, ${ }^{68}$ C.O. Escobar, ${ }^{4,53}$ J. Espadanal, ${ }^{1}$ A. Etchegoyen, ${ }^{11,10}$ P. Facal San Luis, ${ }^{57}$ I. Fajardo Tapia, ${ }^{12}$ H. Falcke $,{ }^{15}, 69$ G. Farrar,${ }^{8}$ A.C. Fauth, ${ }^{53}$ N. Fazzini, ${ }^{4}$ A.P. Ferguson, ${ }^{46}$ B. Fick,${ }^{54}$ A. Filevich,${ }^{11}$ A. Filipcic, ${ }^{70}{ }^{71}$ S. Fliescher, ${ }^{68}$ C.E. Fracchiolla, ${ }^{41}$ E.D. Fraenkel,${ }^{63}$ O. Fratu,${ }^{25}$ U. Fröhlich, ${ }^{24}$ B. Fuchs,${ }^{28}$ R. Gaior,${ }^{20}$

R.F. Gamarra, ${ }^{11}$ S. Gambetta, ${ }^{72}$ B. García,${ }^{61}$ S.T. Garcia Roca, ${ }^{13}$ D. Garcia-Gamez, ${ }^{29}$ D. Garcia-Pinto, ${ }^{19}$

A. Gascon, ${ }^{45}$ H. Gemmeke, ${ }^{26}$ P.L. Ghia, ${ }^{20}$ U. Giaccari, ${ }^{51}$ M. Giller, ${ }^{73}$ H. Glass,${ }^{4}$ M.S. Gold,${ }^{32}$ G. Golup,${ }^{7}$ F. Gomez Albarracin, ${ }^{18}$ M. Gómez Berisso, ${ }^{7}$ P.F. Gómez Vitale, ${ }^{23}$ P. Gonçalves, ${ }^{1}$ D. Gonzalez, ${ }^{21}$ J.G. Gonzalez,${ }^{31}$

B. Gookin, ${ }^{41}$ A. Gorgi,${ }^{2}$ P. Gouffon,${ }^{5}$ E. Grashorn, ${ }^{9}$ S. Grebe,${ }^{15,}{ }^{56}$ N. Griffith,${ }^{9}$ M. Grigat,${ }^{68}$ A.F. Grillo, ${ }^{74}$

Y. Guardincerri, ${ }^{59}$ F. Guarino, ${ }^{14}$ G.P. Guedes, ${ }^{75}$ A. Guzman, ${ }^{12}$ P. Hansen, ${ }^{18}$ D. Harari, ${ }^{7}$ S. Harmsma,${ }^{63,56}$ T.A. Harrison, ${ }^{27}$ J.L. Harton, ${ }^{41}$ A. Haungs, ${ }^{31}$ T. Hebbeker,${ }^{68}$ D. Heck, ${ }^{31}$ A.E. Herve, ${ }^{27}$ C. Hojvat, ${ }^{4}$ N. Hollon, ${ }^{57}$ V.C. Holmes, ${ }^{27}$ P. Homola, ${ }^{40}$ J.R. Hörandel, ${ }^{15}$ A. Horneffer, ${ }^{15}$ P. Horvath, ${ }^{76}$ M. Hrabovský, ${ }^{76,37}$ D. Huber, ${ }^{21}$ T. Huege, ${ }^{31}$ A. Insolia, ${ }^{49}$ F. Ionita, ${ }^{57}$ A. Italiano, ${ }^{49}$ C. Jarne, ${ }^{18}$ S. Jiraskova, ${ }^{15}$ M. Josebachuili, ${ }^{11}$ K. Kadija, ${ }^{17}$ K.H. Kampert, ${ }^{33}$ P. Karhan,${ }^{77}$ P. Kasper ${ }^{4}$ B. Kégl, ${ }^{29}$ B. Keilhauer, ${ }^{31}$ A. Keivani, ${ }^{78}$ J.L. Kelley, ${ }^{15}$ E. Kemp, ${ }^{53}$ R.M. Kieckhafer, ${ }^{54}$ H.O. Klages, ${ }^{31}$ M. Kleifges, ${ }^{26}$ J. Kleinfeller, ${ }^{55,31}$ J. Knapp, ${ }^{44}$ D.-H. Koang, ${ }^{22}$ K. Kotera, ${ }^{57}$ N. Krohm, ${ }^{33}$ O. Krömer, ${ }^{26}$ D. Kruppke-Hansen, ${ }^{33}$ F. Kuehn, ${ }^{4}$ D. Kuempel, ${ }^{24,}{ }^{33}$ J.K. Kulbartz, ${ }^{79}$ N. Kunka, ${ }^{26}$ G. La

Rosa,${ }^{50}$ C. Lachaud,${ }^{6}$ R. Lauer,${ }^{32}$ P. Lautridou, ${ }^{34}$ S. Le Coz ${ }^{22}$ M.S.A.B. Leão, ${ }^{80}$ D. Lebrun, ${ }^{22}$ P. Lebrun, ${ }^{4}$ M.A. Leigui de Oliveira, ${ }^{80}$ A. Letessier-Selvon, ${ }^{20} \mathrm{I}$. Lhenry-Yvon, ${ }^{64} \mathrm{~K}$. Link, ${ }^{21}$ R. López, ${ }^{81}$ A. Lopez Agüera, ${ }^{13}$ K. Louedec, ${ }^{22,29}$ J. Lozano Bahilo, ${ }^{45}$ L. Lu, ${ }^{44}$ A. Lucero, ${ }^{11}$ M. Ludwig,${ }^{21}$ H. Lyberis,${ }^{64}$ C. Macolino, ${ }^{20}$ S. Maldera, ${ }^{2}$

D. Mandat,${ }^{37}$ P. Mantsch, ${ }^{4}$ A.G. Mariazzi, ${ }^{18}$ J. Marin,${ }^{55,2}$ V. Marin,${ }^{34}$ I.C. Maris,${ }^{20}$ H.R. Marquez Falcon, ${ }^{82}$ G. Marsella,${ }^{83}$ D. Martello, ${ }^{51}$ L. Martin,${ }^{34}$ H. Martinez, ${ }^{84}$ O. Martínez Bravo, ${ }^{81}$ H.J. Mathes, ${ }^{31}$ J. Matthews, ${ }^{78,85}$ J.A.J. Matthews, ${ }^{32}$ G. Matthiae ${ }^{38}$ D. Maurel,${ }^{31}$ D. Maurizio,${ }^{52}$ P.O. Mazur, ${ }^{4}$ G. Medina-Tanco,${ }^{12}$ M. Melissas ${ }^{21}$

D. Melo, ${ }^{11}$ E. Menichetti, ${ }^{52}$ A. Menshikov, ${ }^{26}$ P. Mertsch, ${ }^{86}$ C. Meurer, ${ }^{68}$ S. Micanovic, ${ }^{17}$ M.I. Micheletti, ${ }^{87}$

L. Miramonti, ${ }^{48}$ L. Molina-Bueno, ${ }^{45}$ S. Mollerach,${ }^{7}$ M. Monasor,${ }^{57}$ D. Monnier Ragaigne, ${ }^{29}$ F. Montanet ${ }^{22}$ B. Morales ${ }^{12}$ C. Morello, ${ }^{2}$ E. Moreno, ${ }^{81}$ J.C. Moreno, ${ }^{18}$ M. Mostafá, ${ }^{41}$ C.A. Moura ${ }^{80}$ M.A. Muller, ${ }^{53}$ G. Müller,${ }^{68}$ M. Münchmeyer ${ }^{20}$ R. Mussa, ${ }^{52}$ G. Navarra, ${ }^{2, G}$ J.L. Navarro, ${ }^{45}$ S. Navas, ${ }^{45}$ P. Necesal, ${ }^{37}$ L. Nellen, ${ }^{12}$ A. Nelles, ${ }^{15}, 56$ J. Neuser ${ }^{33}$ D. Newton, ${ }^{44}$ P.T. Nhung, ${ }^{67}$ M. Niechciol,${ }^{24}$ L. Niemietz, ${ }^{33}$ N. Nierstenhoefer,${ }^{33}$ D. Nitz, ${ }^{54}$ D. Nosek, ${ }^{77}$

L. Nožka, ${ }^{37}$ M. Nyklicek, ${ }^{37}$ J. Oehlschläger, ${ }^{31}$ A. Olinto, ${ }^{57}$ M. Ortiz, ${ }^{19}$ N. Pacheco, ${ }^{36}$ D. Pakk Selmi-Dei, ${ }^{53}$ M. Palatka, ${ }^{37}$ J. Pallotta, ${ }^{88}$ N. Palmieri, ${ }^{21}$ G. Parente, ${ }^{13}$ E. Parizot,${ }^{6}$ A. Parra,${ }^{13}$ S. Pastor,${ }^{89}$ T. Paul, ${ }^{90}$ M. Pech, ${ }^{37}$ J. Pekala, ${ }^{40}$ R. Pelayo, ${ }^{81}, 13$ I.M. Pepe,${ }^{91}$ L. Perrone,${ }^{83}$ R. Pesce,${ }^{72}$ E. Petermann, ${ }^{92}$ S. Petrera,${ }^{65}$ P. Petrinca,${ }^{38}$ A. Petrolini, ${ }^{72}$ Y. Petrov,${ }^{41}$ C. Pfendner ${ }^{3}$ R. Piegaia, ${ }^{59}$ T. Pierog, ${ }^{31}$ P. Pieroni,${ }^{59}$ M. Pimenta, ${ }^{1}$ V. Pirronello, ${ }^{49}$ M. Platino, ${ }^{11}$ V.H. Ponce,${ }^{7}$ M. Pontz, ${ }^{24}$ A. Porcelli, ${ }^{31}$ P. Privitera, ${ }^{57}$ M. Prouza, ${ }^{37}$ E.J. Quel, ${ }^{88}$ S. Querchfeld, ${ }^{33}$ J. Rautenberg, ${ }^{33}$ O. Ravel, ${ }^{34}$ D. Ravignani, ${ }^{11}$ B. Revenu, ${ }^{34}$ J. Ridky, ${ }^{37}$ S. Riggi, ${ }^{13}$ M. Risse, ${ }^{24}$ P. Ristori, ${ }^{88}$

H. Rivera, ${ }^{48}$ V. Rizi ${ }^{65}$ J. Roberts, ${ }^{8}$ W. Rodrigues de Carvalho, ${ }^{13}$ G. Rodriguez, ${ }^{13}$ J. Rodriguez Martino, ${ }^{55}$ J. Rodriguez Rojo, ${ }^{55}$ I. Rodriguez-Cabo, ${ }^{13}$ M.D. Rodríguez-Frías, ${ }^{36}$ G. Ros ${ }^{36}$ J. Rosado, ${ }^{19}$ T. Rossler,${ }^{76}$ M. Roth,${ }^{31}$ 
B. Rouillé-d'Orfeuil, ${ }^{57}$ E. Roulet, ${ }^{7}$ A.C. Rovero, ${ }^{58}$ C. Rühle, ${ }^{26}$ A. Saftoiu, ${ }^{42}$ F. Salamida, ${ }^{64}$ H. Salazar, ${ }^{81}$ F. Salesa Greus, ${ }^{41}$ G. Salina, ${ }^{38}$ F. Sánchez, ${ }^{11}$ C.E. Santo, ${ }^{1}$ E. Santos, ${ }^{1}$ E.M. Santos, ${ }^{39}$ F. Sarazin, ${ }^{93}$ B. Sarkar, ${ }^{33}$ S. Sarkar, ${ }^{86}$ R. Sato, ${ }^{55}$ N. Scharf, ${ }^{68}$ V. Scherini, ${ }^{48}$ H. Schieler, ${ }^{31}$ P. Schiffer, ${ }^{79,} 68$ A. Schmidt, ${ }^{26}$ O. Scholten, ${ }^{63}$ H. Schoorlemmer, ${ }^{15,56}$ J. Schovancova, ${ }^{37}$ P. Schovánek, ${ }^{37}$ F. Schröder, ${ }^{31}$ S. Schulte, ${ }^{68}$ D. Schuster, ${ }^{93}$ S.J. Sciutto, ${ }^{18}$ M. Scuderi, ${ }^{49}$ A. Segreto, ${ }^{50}$ M. Settimo, ${ }^{24}$ A. Shadkam, ${ }^{78}$ R.C. Shellard, ${ }^{28,66}$ I. Sidelnik, ${ }^{11}$ G. Sigl, ${ }^{79}$ H.H. Silva Lopez, ${ }^{12}$ O. Sima, ${ }^{94}$ A. Smialkowski, ${ }^{73}$ R. Šmída, ${ }^{31}$ G.R. Snow, ${ }^{92}$ P. Sommers, ${ }^{47}$ J. Sorokin, ${ }^{27}$ H. Spinka, ${ }^{95,4}$ R. Squartini, ${ }^{55}$ Y.N. Srivastava, ${ }^{90}$ S. Stanic, ${ }^{71}$ J. Stapleton, ${ }^{9}$ J. Stasielak, ${ }^{40}$ M. Stephan, ${ }^{68}$ A. Stutz, ${ }^{22}$ F. Suarez, ${ }^{11}$ T. Suomijärvi, ${ }^{64}$ A.D. Supanitsky, ${ }^{58}$ T. Šuša, ${ }^{17}$ M.S. Sutherland, ${ }^{78}$ J. Swain, ${ }^{90}$ Z. Szadkowski, ${ }^{73}$ M. Szuba, ${ }^{31}$ A. Tapia, ${ }^{11}$ M. Tartare,${ }^{22}$ O. Tascau, ${ }^{33}$ C.G. Tavera Ruiz, ${ }^{12}$ R. Tcaciuc,${ }^{24}$ D. Tegolo, ${ }^{49}$ N.T. Thao, ${ }^{67}$ D. Thomas, ${ }^{41}$ J. Tiffenberg, ${ }^{59}$ C. Timmermans, ${ }^{56,15}$ W. Tkaczyk, ${ }^{73}$ C.J. Todero Peixoto, ${ }^{62}$ G. Toma, ${ }^{42}$ B. Tomé, ${ }^{1}$ A. Tonachini, ${ }^{52}$ P. Travnicek, ${ }^{37}$ D.B. Tridapalli, ${ }^{5}$ G. Tristram,${ }^{6}$ E. Trovato, ${ }^{49}$ M. Tueros ${ }^{13}$ R. Ulrich, ${ }^{31}$ M. Unger ${ }^{31}$

M. Urban, ${ }^{29}$ J.F. Valdés Galicia, ${ }^{12}$ I. Valiño, ${ }^{13}$ L. Valore, ${ }^{14}$ A.M. van den Berg, ${ }^{63}$ E. Varela, ${ }^{81}$ B. Vargas Cárdenas, ${ }^{12}$ J.R. Vázquez, ${ }^{19}$ R.A. Vázquez, ${ }^{13}$ D. Veberic, ${ }^{71,70}$ V. Verzi, ${ }^{38}$ J. Vicha, ${ }^{37}$ M. Videla, ${ }^{61}$

L. Villaseñor, ${ }^{82}$ H. Wahlberg, ${ }^{18}$ P. Wahrlich, ${ }^{27}$ O. Wainberg, ${ }^{11,10}$ D. Walz, ${ }^{68}$ A.A. Watson, ${ }^{44}$ M. Weber, ${ }^{26}$

K. Weidenhaupt, ${ }^{68}$ A. Weindl, ${ }^{31}$ F. Werner, ${ }^{21}$ S. Westerhoff, ${ }^{3}$ B.J. Whelan, ${ }^{27}$ A. Widom, ${ }^{90}$ G. Wieczorek, ${ }^{73}$

L. Wiencke, ${ }^{93}$ B. Wilczynska, ${ }^{40} \mathrm{H}$. Wilczynski, ${ }^{40} \mathrm{M}$. Will, ${ }^{31} \mathrm{C}$. Williams, ${ }^{57} \mathrm{~T}$. Winchen, ${ }^{68} \mathrm{M}$. Wommer, ${ }^{31}$

B. Wundheiler, ${ }^{11}$ T. Yamamoto, ${ }^{57, d}$ T. Yapici, ${ }^{54}$ P. Younk, ${ }^{24,}, 96$ G. Yuan, ${ }^{78}$ A. Yushkov, ${ }^{13}$ B. Zamorano, ${ }^{45}$ E. Zas, ${ }^{13}$

D. Zavrtanik, ${ }^{71,70}$ M. Zavrtanik, ${ }^{70,71}$ I. Zaw, ${ }^{8, \text { e A. Zepeda, }}{ }^{84}$ Y. Zhu, ${ }^{26}$ M. Zimbres Silva, ${ }^{33,53}$ and M. Ziolkowski ${ }^{24}$

(The Pierre Auger Collaboration)

${ }^{1}$ LIP and Instituto Superior Técnico, Technical University of Lisbon, Portugal

${ }^{2}$ Istituto di Fisica dello Spazio Interplanetario (INAF),

Università di Torino and Sezione INFN, Torino, Italy

${ }^{3}$ University of Wisconsin, Madison, WI, USA

${ }^{4}$ Fermilab, Batavia, IL, USA

${ }^{5}$ Universidade de São Paulo, Instituto de Física, São Paulo, SP, Brazil

${ }^{6}$ Laboratoire AstroParticule et Cosmologie (APC),

Université Paris 7, CNRS-IN2P3, Paris, France

${ }^{7}$ Centro Atómico Bariloche and Instituto Balseiro (CNEA-UNCuyo-CONICET), San Carlos de Bariloche, Argentina

${ }^{8}$ New York University, New York, NY, USA

${ }^{9}$ Ohio State University, Columbus, OH, USA

${ }^{10}$ Universidad Tecnológica Nacional - Facultad Regional Buenos Aires, Buenos Aires, Argentina

${ }^{11}$ Instituto de Tecnologías en Detección y Astropartículas (CNEA, CONICET, UNSAM), Buenos Aires, Argentina

${ }^{12}$ Universidad Nacional Autonoma de Mexico, Mexico, D.F., Mexico

${ }^{13}$ Universidad de Santiago de Compostela, Spain

${ }^{14}$ Università di Napoli "Federico II" and Sezione INFN, Napoli, Italy

${ }^{15}$ IMAPP, Radboud University Nijmegen, Netherlands

${ }^{16}$ University of Wisconsin, Milwaukee, WI, USA

${ }^{17}$ Rudjer Boškovic Institute, 10000 Zagreb, Croatia

${ }^{18}$ IFLP, Universidad Nacional de La Plata and CONICET, La Plata, Argentina

${ }^{19}$ Universidad Complutense de Madrid, Madrid, Spain

${ }^{20}$ Laboratoire de Physique Nucléaire et de Hautes Energies (LPNHE), Universités Paris 6 et Paris 7, CNRS-IN2P3, Paris, France

${ }^{21}$ Karlsruhe Institute of Technology - Campus South - Institut für Experimentelle Kernphysik (IEKP), Karlsruhe, Germany

${ }^{22}$ Laboratoire de Physique Subatomique et de Cosmologie (LPSC),

Université Joseph Fourier, INPG, CNRS-IN2P3, Grenoble, France

${ }^{23}$ Observatorio Pierre Auger and Comisión Nacional de Energía Atómica, Malargüe, Argentina

${ }^{24}$ Universität Siegen, Siegen, Germany

${ }^{25}$ University Politehnica of Bucharest, Romania

${ }^{26}$ Karlsruhe Institute of Technology - Campus North - Institut für Prozessdatenverarbeitung und Elektronik, Karlsruhe, Germany ${ }^{27}$ University of Adelaide, Adelaide, S.A., Australia

${ }^{28}$ Centro Brasileiro de Pesquisas Fisicas, Rio de Janeiro, RJ, Brazil

${ }^{29}$ Laboratoire de l'Accélérateur Linéaire (LAL), Université Paris 11, CNRS-IN2P3, Orsay, France

${ }^{30}$ Universidade Estadual do Sudoeste da Bahia, Vitoria da Conquista, BA, Brazil

${ }^{31}$ Karlsruhe Institute of Technology - Campus North - Institut für Kernphysik, Karlsruhe, Germany

${ }^{32}$ University of New Mexico, Albuquerque, NM, USA

${ }^{33}$ Bergische Universität Wuppertal, Wuppertal, Germany

${ }^{34}$ SUBATECH, École des Mines de Nantes, CNRS-IN2P3, Université de Nantes, Nantes, France

${ }^{35}$ Max-Planck-Institut für Radioastronomie, Bonn, Germany

${ }^{36}$ Universidad de Alcalá, Alcalá de Henares (Madrid), Spain

${ }^{37}$ Institute of Physics of the Academy of Sciences of the Czech Republic, Prague, Czech Republic 
${ }^{38}$ Università di Roma II "Tor Vergata" and Sezione INFN, Roma, Italy

${ }^{39}$ Universidade Federal do Rio de Janeiro, Instituto de Física, Rio de Janeiro, RJ, Brazil

${ }^{40}$ Institute of Nuclear Physics PAN, Krakow, Poland

${ }^{41}$ Colorado State University, Fort Collins, CO, USA

42 'Horia Hulubei' National Institute for Physics and Nuclear Engineering, Bucharest-Magurele, Romania ${ }^{43}$ Colorado State University, Pueblo, CO, USA

${ }^{44}$ School of Physics and Astronomy, University of Leeds, United Kingdom

${ }^{45}$ Universidad de Granada 83 C.A.F.P.E., Granada, Spain

${ }^{46}$ Case Western Reserve University, Cleveland, OH, USA

${ }^{47}$ Pennsylvania State University, University Park, PA, USA

${ }^{48}$ Università di Milano and Sezione INFN, Milan, Italy

${ }^{49}$ Università di Catania and Sezione INFN, Catania, Italy

${ }^{50}$ Istituto di Astrofisica Spaziale e Fisica Cosmica di Palermo (INAF), Palermo, Italy

${ }^{51}$ Dipartimento di Fisica dell'Università del Salento and Sezione INFN, Lecce, Italy

${ }^{52}$ Università di Torino and Sezione INFN, Torino, Italy

${ }^{53}$ Universidade Estadual de Campinas, IFGW, Campinas, SP, Brazil

${ }^{54}$ Michigan Technological University, Houghton, MI, USA

${ }^{55}$ Observatorio Pierre Auger, Malargüe, Argentina

${ }^{56}$ Nikhef, Science Park, Amsterdam, Netherlands

${ }^{57}$ University of Chicago, Enrico Fermi Institute, Chicago, IL, USA

${ }^{58}$ Instituto de Astronomía y Física del Espacio (CONICET-UBA), Buenos Aires, Argentina

${ }^{59}$ Departamento de Física, FCEyN, Universidad de Buenos Aires y CONICET, Argentina

${ }^{60}$ Universidade Federal Fluminense, EEIMVR, Volta Redonda, RJ, Brazil

${ }^{61}$ National Technological University, Faculty Mendoza (CONICET/CNEA), Mendoza, Argentina

${ }^{62}$ Universidade de São Paulo, Instituto de Física, São Carlos, SP, Brazil

${ }^{63}$ Kernfysisch Versneller Instituut, University of Groningen, Groningen, Netherlands

${ }^{64}$ Institut de Physique Nucléaire d'Orsay (IPNO),

Université Paris 11, CNRS-IN2P3, Orsay, France

${ }^{65}$ Università dell'Aquila and INFN, L'Aquila, Italy

${ }^{66}$ Pontifícia Universidade Católica, Rio de Janeiro, RJ, Brazil

${ }^{67}$ Institute for Nuclear Science and Technology (INST), Hanoi, Vietnam

${ }^{68}$ RWTH Aachen University, III. Physikalisches Institut A, Aachen, Germany

${ }^{69}$ ASTRON, Dwingeloo, Netherlands

${ }^{70}$ J. Stefan Institute, Ljubljana, Slovenia

${ }^{71}$ Laboratory for Astroparticle Physics, University of Nova Gorica, Slovenia

${ }^{72}$ Dipartimento di Fisica dell'Università and INFN, Genova, Italy

${ }^{73}$ University of Lódz, Lódz, Poland

${ }^{74}$ INFN, Laboratori Nazionali del Gran Sasso, Assergi (L'Aquila), Italy

${ }^{75}$ Universidade Estadual de Feira de Santana, Brazil

${ }^{76}$ Palacky University, RCPTM, Olomouc, Czech Republic

${ }^{77}$ Charles University, Faculty of Mathematics and Physics,

Institute of Particle and Nuclear Physics, Prague, Czech Republic

${ }^{78}$ Louisiana State University, Baton Rouge, LA, USA

${ }^{79}$ Universität Hamburg, Hamburg, Germany

${ }^{80}$ Universidade Federal do ABC, Santo André, SP, Brazil

${ }^{81}$ Benemérita Universidad Autónoma de Puebla, Puebla, Mexico

${ }^{82}$ Universidad Michoacana de San Nicolas de Hidalgo, Morelia, Michoacan, Mexico

${ }^{83}$ Dipartimento di Ingegneria dell'Innovazione dell'Università del Salento and Sezione INFN, Lecce, Italy

${ }^{84}$ Centro de Investigación y de Estudios Avanzados del IPN (CINVESTAV), México, D.F., Mexico

${ }^{85}$ Southern University, Baton Rouge, LA, USA

${ }^{86}$ Rudolf Peierls Centre for Theoretical Physics, University of Oxford, Oxford, United Kingdom

${ }^{87}$ Instituto de Física de Rosario (IFIR) - CONICET/U.N.R. and Facultad

de Ciencias Bioquímicas y Farmacéuticas U.N.R., Rosario, Argentina

${ }^{88}$ Centro de Investigaciones en Láseres y Aplicaciones, CITEFA and CONICET, Argentina

${ }^{89}$ Instituto de Física Corpuscular, CSIC-Universitat de València, Valencia, Spain

${ }^{90}$ Northeastern University, Boston, MA, USA

${ }^{91}$ Universidade Federal da Bahia, Salvador, BA, Brazil

${ }^{92}$ University of Nebraska, Lincoln, NE, USA

${ }^{93}$ Colorado School of Mines, Golden, CO, USA

${ }^{94}$ University of Bucharest, Physics Department, Romania

${ }^{95}$ Argonne National Laboratory, Argonne, IL, USA

${ }^{96}$ Los Alamos National Laboratory, Los Alamos, NM, USA 


\begin{abstract}
The Surface Detector of the Pierre Auger Observatory is sensitive to neutrinos of all flavours above $0.1 \mathrm{EeV}$. These interact through charged and neutral currents in the atmosphere giving rise to extensive air showers. When interacting deeply in the atmosphere at nearly horizontal incidence, neutrinos can be distinguished from regular hadronic cosmic rays by the broad time structure of their shower signals in the water-Cherenkov detectors. In this paper we present for the first time an analysis based on down-going neutrinos. We describe the search procedure, the possible sources of background, the method to compute the exposure and the associated systematic uncertainties. No candidate neutrinos have been found in data collected from 1 January 2004 to 31 May 2010. Assuming an $E^{-2}$ differential energy spectrum the limit on the single flavour neutrino is $E^{2} \mathrm{~d} N / \mathrm{d} E<$ $1.74 \times 10^{-7} \mathrm{GeV} \mathrm{cm}^{-2} \mathrm{~s}^{-1} \mathrm{sr}^{-1}$ at $90 \%$ C.L. in the energy range $1 \times 10^{17} \mathrm{eV}<E<1 \times 10^{20} \mathrm{eV}$.
\end{abstract}

PACS numbers: 95.55.Vj, 95.85.Ry, 98.70.Sa

\section{INTRODUCTION}

Neutrinos play a key role in the understanding of the origin of ultra high energy cosmic rays (UHECRs). Their observation should open a new window to the universe since they can give information on regions that are otherwise hidden by large amounts of matter in the field of view. Moreover, neutrinos are not deviated by magnetic fields and would point back to their sources.

In the EeV range, neutrinos are expected to be produced in the same sources where UHECRs are thought to be accelerated, as well as during the propagation of UHECRs through the cosmic microwave background (CMB) radiation [1]. The latter are called cosmogenic neutrinos and their presence is expected if the UHECRs above the spectral cut-off reported in [2] contain a significant fraction of protons [3 8 ].

There are many current programs to search for high energy neutrinos with dedicated experiments [9 11]. Although the primary goal of the Pierre Auger Observatory Surface (SD) and Fluorescence Detectors (FD) is to detect UHECRs, UHE neutrinos (UHE $\nu$ s) can also be identified and limits to the diffuse flux of $\mathrm{UHE} \nu \mathrm{s}$ in the EeV range and above have been set using earlier Auger data [12 14]. Earth-skimming $\tau$ neutrinos are expected to be observed through the detection of showers induced by the decay of emerging $\tau$ leptons which are created by $\nu_{\tau}$ interactions in the Earth [15]. Using this mechanism for data collected from 1 January 2004 until 30 April 2008, an upper limit was set: $E_{\nu}^{2} d N_{\nu_{\tau}} /$ $d E_{\nu}<6_{-3}^{+3} \times 10^{-8} \mathrm{GeV} \mathrm{cm}^{-2} \mathrm{~s}^{-1} \mathrm{sr}^{-1}$ at $90 \% \mathrm{CL}$ for each neutrino flavour [16]. The SD of the Pierre Auger Observatory has also been shown to be sensitive to "downgoing" neutrinos of all flavours interacting in the atmosphere or in the mountains surrounding the SD, and inducing a shower close to the ground [14, 17, 18]. In this paper we present an analysis based on down-going neutrinos and place a competitive limit on the all-flavour

\footnotetext{
a now at University of Maryland.

b now at Universit de Lausanne.

c Deceased

d now at Konan University, Kobe, Japan.

e now at NYU Abu Dhabi.
}

diffuse neutrino flux using data from 1 January 2004 until 31 May 2010.

The main challenge in detecting UHE neutrinos with the Pierre Auger Observatory is to identify a neutrinoinduced shower in the background of showers initiated by UHECRs, possibly protons or heavy nuclei [19] and, in a much smaller proportion, even photons [20].

The identification of $\nu$-induced showers is illustrated in Fig. 1. If the incidence is nearly horizontal, "old" showers induced in the upper atmosphere by protons, nuclei or photons have a thin and flat front at ground level, containing only high energy muons and their radiative and decay products, concentrated within a few tens of nanoseconds. On the other hand, "young" showers, induced by neutrinos at a low altitude, have a thick, curved front with a significant electromagnetic component spread in time over hundreds of nanoseconds, specially in their earlier part that traverses less atmosphere. In this work, to obtain an unambiguous identification of neutrinos, we select showers with zenith angle $\theta>75^{\circ}$ and we apply criteria to ensure a deep interaction. Using less inclined showers is in principle possible, but will require a better control of the various sources of background.

The method was tuned using data taken at the SD in the period from 1 January 2004 until 31 October 2007. A blind scan over the data collected in the remaining period, i.e., from 1 November 2007 until 31 May 2010 reveals no candidates and we place a stringent limit on the diffuse flux of UHE neutrinos.

For that purpose, we calculate the probability for a shower, produced deeply in the atmosphere, to trigger the SD and to be identified as a neutrino candidate. This probability depends on the neutrino flavour and type of interaction - charged current (CC) or neutral current (NC) - and is also a function of neutrino energy $E_{\nu}$, incident zenith angle $\theta$, and atmospheric interaction depth. From these identification probabilities we calculate the exposure of the SD to deep inclined neutrino showers. We give an estimate of the systematic uncertainties on the diffuse neutrino flux limit, and discuss the implications of our observations for models of UHE neutrino production. 


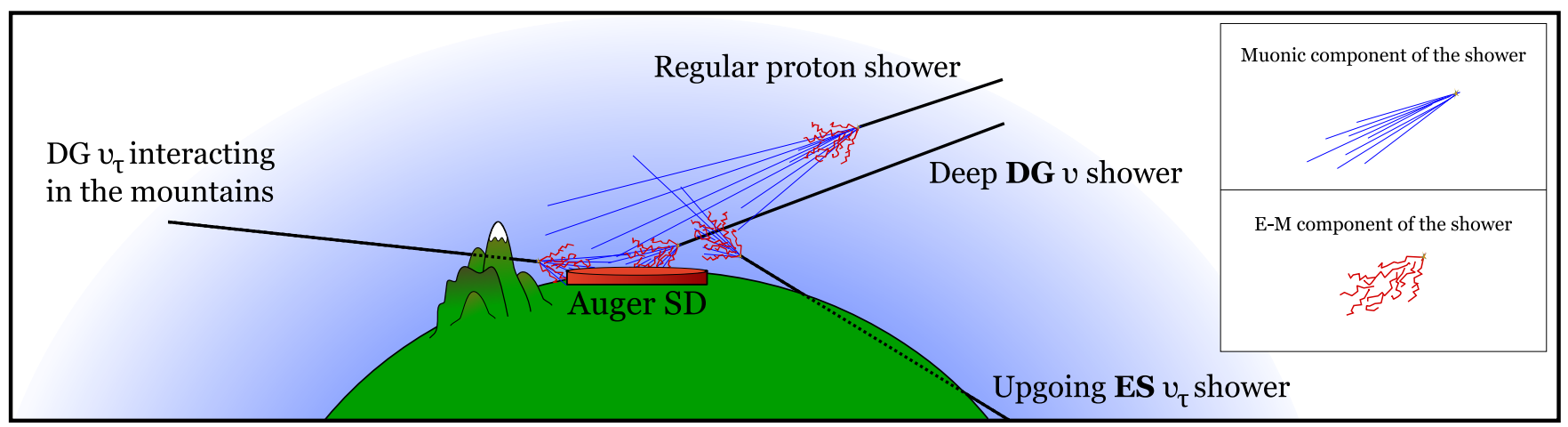

FIG. 1. Pictorial representation of the different types of showers induced by protons, heavy nuclei and "down-going" (DG) as well as "Earth-skimming" (ES) neutrinos. The search for down-going showers initiated deep in the atmosphere is the subject of this work.

\section{THE PIERRE AUGER OBSERVATORY}

The Pierre Auger Observatory is a hybrid detector located in Malargüe, Mendoza, Argentina [21]. It consists of an array of particle detectors 22] and a set of fluorescence telescopes [23] at four sites that provide a unique cross calibration capability.

The SD is spread over a surface of $\sim 3000 \mathrm{~km}^{2}$ at an altitude of $\sim 1400 \mathrm{~m}$ above sea level. This corresponds to an average vertical atmospheric depth above ground of $X_{\text {ground }}=880 \mathrm{~g} \mathrm{~cm}^{-2}$. The slant depth $D$ is the total grammage traversed by a shower measured from ground in the direction of the incoming primary particle. In the flat-Earth approximation $D=\left(X_{\text {ground }}-X_{\text {int }}\right) / \cos \theta$, where $X_{\text {int }}$ is the interaction depth and $\theta$ the zenith angle. For very inclined showers the curvature of the atmosphere is taken into account.

The four fluorescence sites are located at the perimeter of the surface array viewing the atmosphere above it 23]. In this work only data collected with the SD of the Pierre Auger Observatory are used to search for down-going neutrinos.

\section{A. The Surface Detector}

Since the beginning of its operation for physics analysis, in January 2004, the SD array has grown steadily and it has been recording an increasing amount of data. It consists of $\sim 1660$ detector units (water-Cherenkov stations) regularly spaced in a triangular grid of side 1.5 $\mathrm{km}$. Each detector unit is a cylindrical polyethylene tank of $3.6 \mathrm{~m}$ diameter and $1.2 \mathrm{~m}$ height containing 12,000 liters of purified water. The top surface has three photomultiplier tubes (PMTs) in optical contact with the water in the tank. The PMT signals are sampled by flash analog digital converters (FADC) with a frequency of $40 \mathrm{MHz}$. Each surface detector is regularly monitored and calibrated in units of vertical equivalent muons (VEM) corresponding to the signal produced by a $\mu$ traversing the tank vertically and through its center [24]. The surface stations transmit information by radio links to the Central Data Acquisition System (CDAS) located in Malargüe. The PMTs, local processor, GPS receiver, and the radio system are powered by batteries regulated by solar panels. Once installed, the local stations work continuously without external intervention.

\section{B. The trigger}

A local trigger selects signals, either with a high peak value, or with a long duration. The second condition favours stations hit in the early stage of the shower development (moderately inclined or deeply induced showers). The global trigger requires either 4 stations satisfying one of the conditions, or 3 stations satisfying the second one, in a compact configuration (see 25] for more details).

With the complete array, the global trigger rate is about two events per minute, one half being actual shower events with median energy of $3 \times 10^{17} \mathrm{eV}$.

\section{SIMULATION OF NEUTRINO INTERACTIONS, INDUCED SHOWERS AND THE RESPONSE OF THE SURFACE DETECTOR.}

Monte Carlo simulations of neutrino-induced showers are used to establish identification criteria and to compute the acceptance of the SD to UHE $\nu$ s. The whole simulation chain is divided in three stages:

1. High energy processes:

- The $\nu$-nucleon interaction is simulated with HERWIG 26].

- In the case of $\nu_{\tau} \mathrm{CC}$ interactions, the $\tau$ lepton propagation is simulated with a dedicated code and its decay (when necessary) with TAUOLA [27]. 
2. The shower development in the atmosphere is processed by AIRES [28].

3. The Surface Detector simulation is performed with the Offline software [29].

In the next subsections we discuss each stage in detail.

\section{A. Neutrino interaction}

HERWIG is a general-purpose event generator for highenergy processes, with particular emphasis on the detailed simulation of QCD parton showers. Here it is used to compute the fraction of the primary energy that goes into the hadronic vertex and to provide the secondary particles produced for both charged (CC) and neutral current (NC) interactions (see Fig. 2 for a summary of all the channels considered in this work).

The energy carried by the hadronic jet is always converted into a shower which could be seen by the SD. In addition, the energy of the lepton produced in a $\mathrm{CC}$ interaction may be totally or partially visible. An electron is promptly converted into an electromagnetic shower. A $\tau$ at $\mathrm{EeV}$ energies has a decay length of $\sim 50 \mathrm{~km}$ and may decay before reaching the ground producing a secondary shower that can be detected (so called "double bang" event). On the other hand, it is very unlikely that a high energy muon will produce a detectable shower, so its interaction and/or decay are not simulated. For all channels and neutrino flavours a set of primary $\nu$ interactions is constructed from a grid of incoming neutrino energies, zenith angles and interaction depths. In "double bang" events the decay products of the $\tau$ lepton are generated by TAUOLA. The energies and momenta of the secondary particles are then injected into the program AIRES to generate the atmospheric cascade.

\section{B. Down-going neutrinos interacting in the mountains}

In addition to the interactions in the atmosphere, we also take into account the possibility of $\tau$ neutrino interactions within the mountains around the Pierre Auger Observatory (mainly the Andes located to the northwest of the array), producing a hadronic jet and a $\tau$ lepton. The hadronic or electromagnetic showers produced by neutrinos of any flavour are absorbed either in the rock itself, or in the few ten kilometers of atmosphere between the mountains and the Auger array, and may be neglected. So only showers induced by the decay of the $\tau \mathrm{s}$ may be seen. In other terms, this process is exactly equivalent to the "Earth-skimming" mechanism, but it is included in this study because such showers are going downwards.

The topography surrounding the SD of the Auger Observatory is accounted for using a digital elevation map [30]. For the Auger site, the line of sight intercepting the mountains corresponds only to zenith angles very close to the horizon $\left(\theta>89^{\circ}\right)$. Even though the solid angle is much smaller than for showers with $\theta>75^{\circ}$, this mechanism is still relevant because mountains are much more massive. It is simulated in the same way as the "double bang" process, accounting in addition for energy loss of the $\tau$ lepton in the rock [31].

\section{Detector simulation}

To avoid excessively long computing times AIRES uses the standard thinning procedure [32] consisting in following only some branches in the tree of interactions in the atmosphere. Weights are attributed to the surviving branches, obtaining a representative set of particles at any stage, especially at ground level. The first step in the detector response simulation is to regenerate a fair sample of the particles expected in each station from the thinned output of AIRES. This unthinning procedure is detailed in [33]. Each particle reaching a surface detector station is injected in the station, and the amount of Cherenkov light produced in water calculated with GEANT4 34]. The FADC traces of the PMT signals are simulated using the Offline framework [29]. The total signal due to the particles entering the station, as well as several quantities characterizing the FADC trace which will be relevant for neutrino identification (see below) are then calculated. The local and global trigger conditions are applied in the same way as for real data.

\section{INCLINED EVENT SELECTION AND RECONSTRUCTION}

Events occurring during periods of data acquisition instabilities 25] are excluded. After a "trace cleaning" procedure removing the accidental signals (mainly atmospheric muons), the start times of the signals in the stations are requested to be compatible with a plane shower front moving at speed $c$. If this condition is not fulfilled using all stations included in the global trigger, an iterative procedure removes stations until a satisfactory configuration is found with at least four stations. Otherwise the event is rejected. The angle between a vertical axis and the perpendicular direction to this plane is the reconstructed zenith angle $\theta_{\text {rec }}$ of the shower. Nearly horizontal showers are selected by requiring $\theta_{\text {rec }}>75^{\circ}$. In some cases a non-inclined event, produced by detector fluctuations or two independent showers arriving close in time (less that $60 \mathrm{~ns}$ ), may be incorrectly reconstructed as inclined. To remove these events we also compute the apparent speed of propagation of the trigger between every pair of stations $\left(V_{i j}\right)$ and the average speed of the event $(\langle V\rangle)$, as in 16]. Genuine inclined showers have a "footprint" (configuration of the stations) elongated in the direction of arrival (left panel of Fig. 31). The appar- 


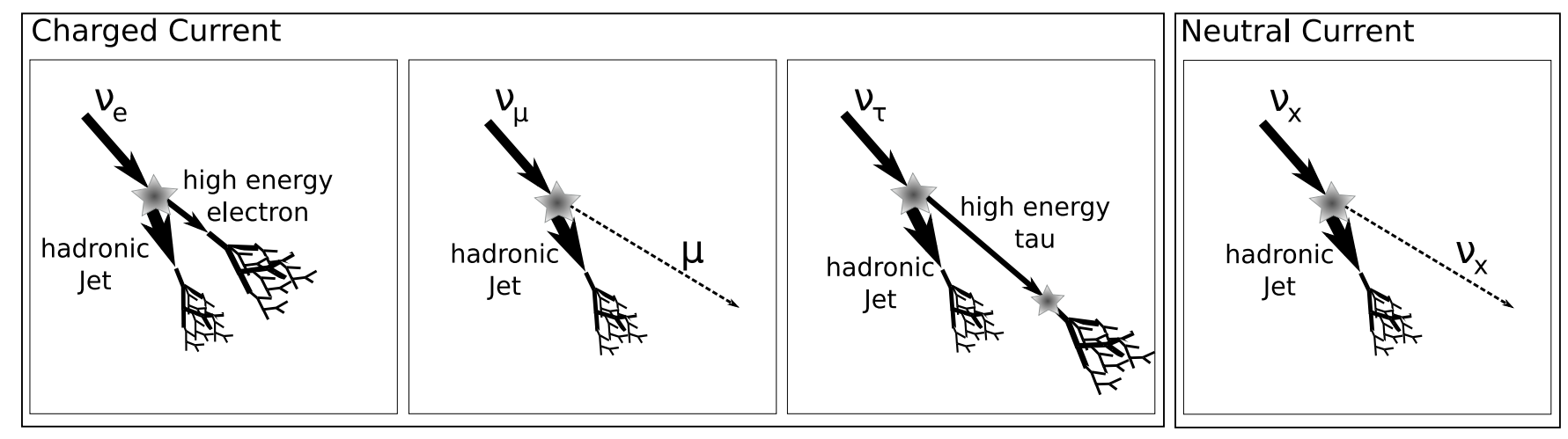

FIG. 2. Different types of atmospheric showers induced by neutrinos.

ent speed of propagation of the signal, along the major axis of the footprint, is concentrated around the speed of light $c$.

Under the plane front approximation, the zenith angle is $\simeq \arcsin (c /\langle V\rangle)$. In Fig. 4 we show the distribution of $\langle V\rangle$ for events with $\theta_{\text {rec }}>75^{\circ}$ acquired between 1 January 2004 and 31 October 2007. The shaded region corresponds to misreconstructed or low quality events (see right panel of Fig. 3 for an example). To remove these events we optimized a set of quality cuts using a MC sample of 5000 regular inclined showers initiated by hadrons near the top of the atmosphere: $\langle V\rangle$ is required to be less than $0.313 \mathrm{~m} \mathrm{~ns}^{-1}$, with a relative spread smaller than $0.08 \%$. Also, the "footprint" is required to be elongated: $L / W>3$, where $L$ and $W$ are the length and the width (eigenvalues of the inertia tensor, as defined in [16]). These cuts reject only $10 \%$ of genuine inclined showers.

For events where all stations are aligned along one of the directions of the array, $\theta_{\text {rec }}$ cannot be computed and we rely on the average speed of the event, $\langle V\rangle$. These "in-line" events are of great importance since the Monte Carlo simulations show that low energy neutrinos $\left(\lesssim 10^{18}\right.$ $\mathrm{eV}$ ) typically present this type of configuration in the SD.

There is an additional requirement for events constituted by an in-line event plus a non-aligned station (a non-aligned event that would become in-line by removing just one station). This kind of spatial configuration is particularly prone to bad reconstruction if the nonaligned station was triggered by accidental muons not belonging to the shower front. To avoid this problem we also reconstruct the in-line event obtained by the removal of the non-aligned station and require it to have mean ground speed compatible with a zenith angle larger than $75^{\circ}$.

\section{IDENTIFICATION OF NEUTRINO CANDIDATES}

For this analysis, the whole data period (1 Jan 04 31 May 10), was divided into two separate samples. Selected events recorded between 1 Jan 04 and 31 Oct 07 (equivalent to $\sim 1.4$ years of a complete SD array working continuosly) constitute the "training" sample, used to develop and optimize the neutrino identification algorithms. Data collected between 1 Nov 07 and 31 May 10 (equivalent to $\sim 2$ yr of the full array), constitute the "search" sample. These latter events were not processed before the final tuning of the algorithms defining the neutrino identification criteria.

\section{A. Discrimination of neutrinos from hadronic showers}

Neutrinos, unlike protons and heavier nuclei, can generate showers initiated deeply into the atmosphere. The main signature of these deep showers in the SD is a significant electromagnetic (EM) component spread in time over hundreds of nanoseconds, especially in the region on the ground at which the shower arrives earlier (see Fig. 5). On the other hand, hadron-induced showers start high in the atmosphere, their electromagnetic component is fully absorbed and only high energy muons and their radiative and decay products reach the surface, concentrated within a few tens of nanoseconds.

We identify stations reached by wide EM-rich shower fronts via their Area-over-Peak ratio (AoP), defined as the ratio of the integral of the FADC trace to its peak value, normalized to 1 for the average signal produced by a single muon. In background horizontal showers the muons and their electromagnetic products are concentrated within a short time interval, so their AoP is close to 1 . In the first stations hit by a deep inclined shower, it is typically between 3 and 5 (see left panel of Fig. 6).

To quantify the distinctive features of hadronic and deeply penetrating showers induced by neutrinos at large 

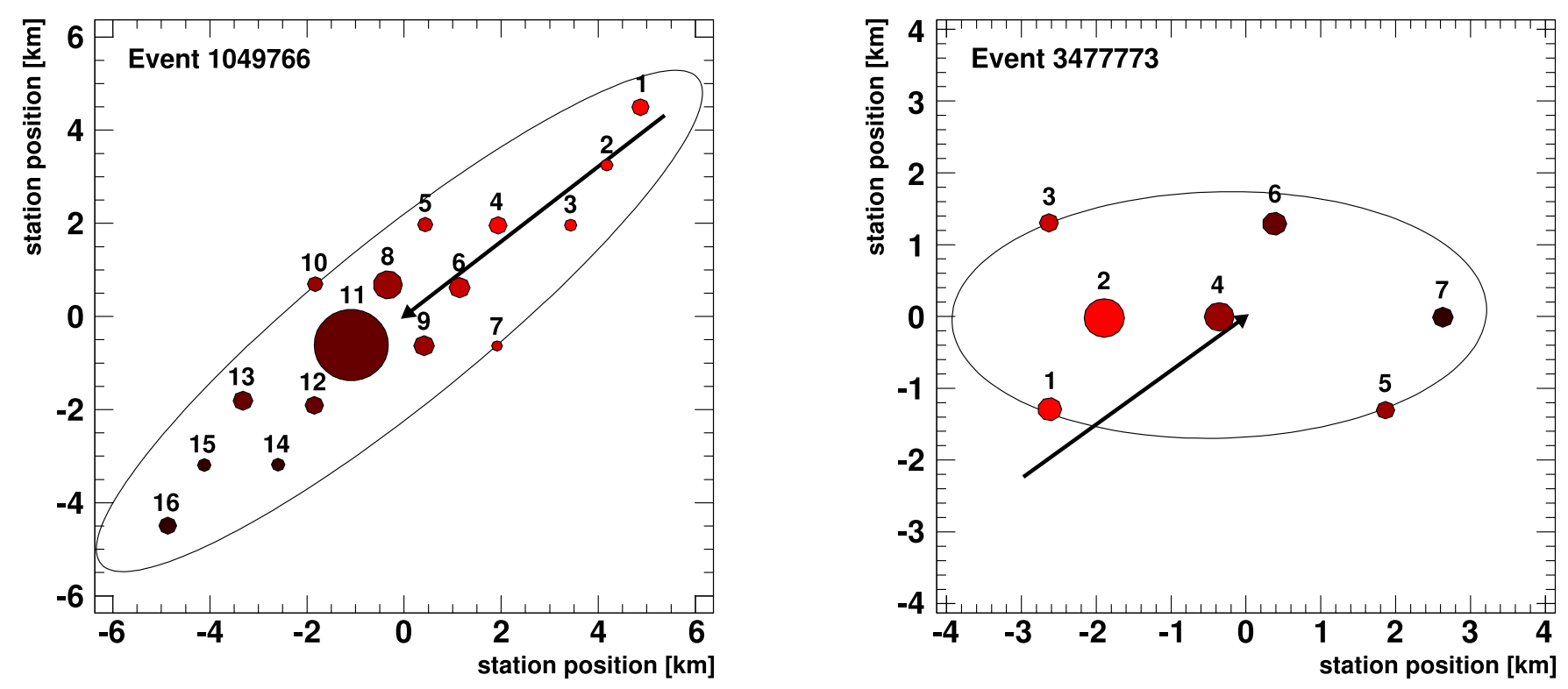

FIG. 3. Left panel: Event produced by a nearly horizontal shower $\left(\theta_{\text {rec }}=80^{\circ}\right)$. The footprint (ellipse) is elongated along the reconstructed direction of arrival (arrow). Right panel: a non-inclined event with $\theta_{r e c}=79^{\circ}$. The major axis of the footprint and the reconstructed direction of arrival do not point in the same direction. Close inspection of the event suggests that stations 3 and 5 are accidental and corrupt the reconstruction. The numbers indicate the triggering order of the stations.

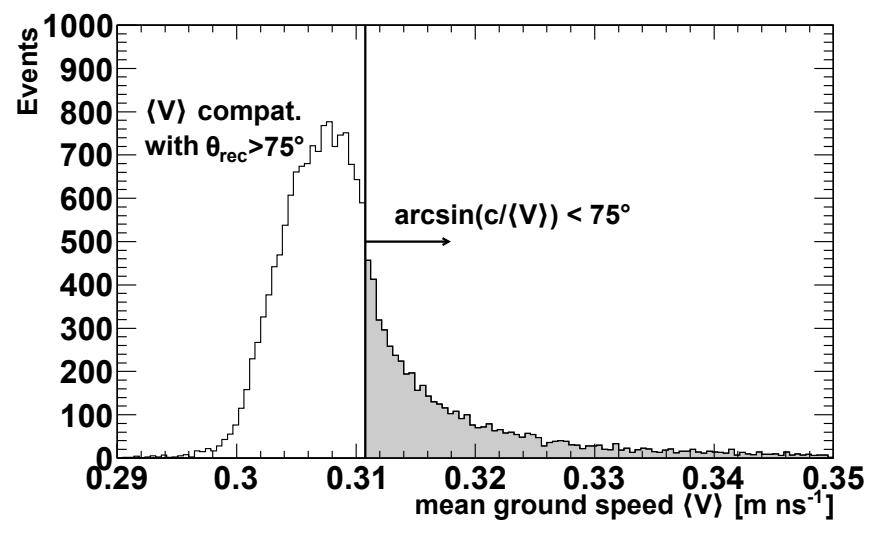

FIG. 4. Distribution of the mean ground speed of the signal for events with $\theta_{\text {rec }}>75^{\circ}$ acquired between 1 January 2004 and 31 October 2007.

zenith angle, improve the separation between the samples and enhance the efficiency, while keeping a simple physical interpretation of the identification process, we choose a multivariate technique known as the Fisher discriminant method [35]. To tune it we used as a "signal" sample the Monte Carlo simulations - exclusively composed of neutrino-induced showers - and as "background" the training sample introduced above - overwhelmingly, if not totally, constituted of nucleonic showers. We use real data to train the Fisher discrimination method, instead of simulations of hadronic showers, for two main reasons:

- the composition of the primary flux is not known,

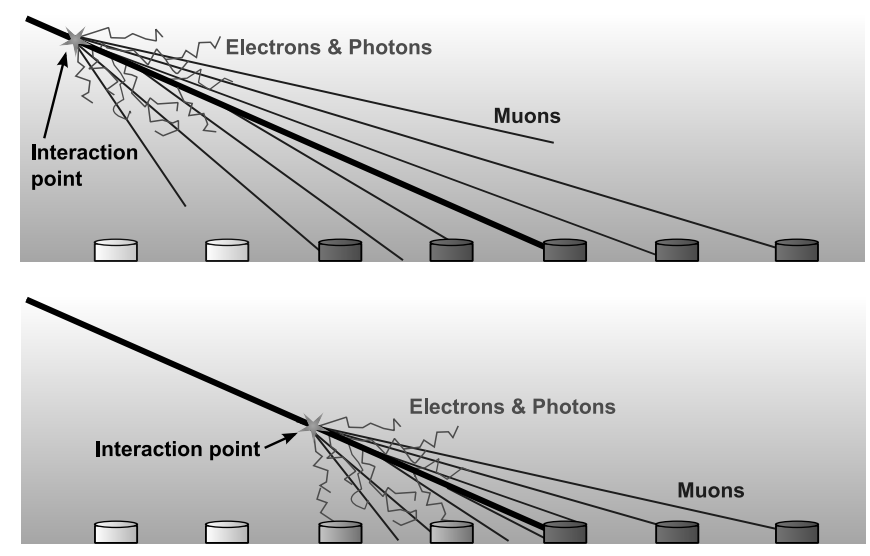

FIG. 5. Upper panel: sketch of an inclined shower induced by a hadron interacting high in the atmosphere. The EM component is absorbed and only the muons reach the detector. Lower panel: deep inclined shower. Its early region has a significant EM component at the detector level.

and moreover the interaction models used to simulate hadronic showers may bias some features of the tail of the distributions of the observables used in this analysis.

- the detector simulation may not account for all possible detector defects or fluctuations that may contribute to the background to ultra-high energy neutrinos, while the real data contain all of them, including those which are not well known, or even 

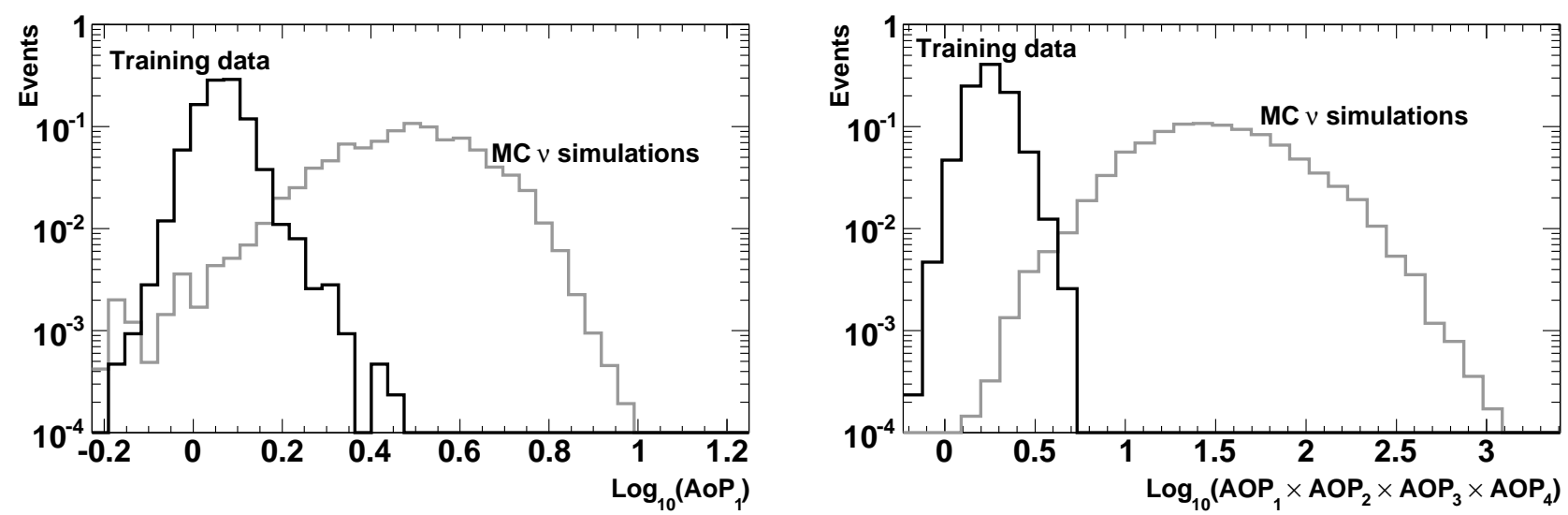

FIG. 6. Distributions of the AoP of the earliest station (left) and the product of the first four AoP (right) in background (real events in the training sample) and simulated $\nu_{e} \mathrm{CC}$ events. There is a clear separation between both samples indicating that the AoP of the early stations is a good discrimination observable to be used in the Fisher method. See text for more details.

not yet diagnosed.

Note that, since we apply a statistical method for the discrimination, the use of real data as a background sample does not imply that we assume it contains no neutrinos, but just that, if any, they constitute a small fraction of the total recorded events.

After training the Fisher method, a good discrimination is found when using the following ten variables 14]: the AoP of the four earliest triggered stations in each event, their squares, their product, and a global early-late asymmetry parameter of the event. We include the square of the AoP because when the distribution of the input variables is not gaussian the addition of a non-linear combination of them improves the discrimination power [36]. The product of the AoP of the earliest four stations in the event aims at minimizing the relative weight of an accidentally large AoP produced, for instance, by a single muon which does not belong to the shower front arriving at a station before or after the shower itself. This variable is also a very good discriminator as shown in the right panel of Fig. 6. The early-late asymmetry parameter is a global observable of the event defined as the difference between the mean AoP of the earliest and latest stations in the event. We have checked in simulations that neutrino-induced events typically have an asymmetry parameter larger than proton or nucleus-induced showers [14]. Finally, the addition of other observables characterizing the time spread of the signals, such as the rise-time (between $10 \%$ and $50 \%$ of the integrated signal) or the fall-time (between $50 \%$ and $90 \%$ ), or including local observables of the stations that trigger last in the event, do not bring about significant improvements in the discrimination.

As the shower front is broader at larger distance from the core for both young and old showers, the discrimination is better when splitting the samples according to the multiplicity $N$ (number of selected stations). A Fisher discriminant was built separately for $4 \leq N \leq 6$, $7 \leq N \leq 11$ and $N \geq 12$. Left panel of Fig. 7 shows the excellent separation achieved for events in each of the 3 sub-samples.

Once the Fisher discriminant $\mathcal{F}$ is defined, one has to choose a threshold value that separates neutrino candidates from regular hadronic showers. Because the predictions of the neutrino detection rates are very low, we want to keep the expected rate of background events incorrectly classified as neutrinos well below any detectable signal: in practice, we wish it to be less than one event for each multiplicity subsample within the expected 20-year lifetime of the Auger Observatory.

The training period was used to produce a reasonable prediction of the background. We observe that the tail of the background distribution of $\mathcal{F}$ is consistent with an exponential shape. In this way, we produced a fit to the distribution of $\mathcal{F}$ for the training data in the $[1 \sigma, 3 \sigma]$ region, where $\sigma$ is the RMS of the training sample. This procedure is illustrated in Fig. 7. We then extrapolated it to find the cuts corresponding to 1 event per 1, 20 or 100 years on the full array. The validity of the extrapolation is not guaranteed, but some physical arguments support an exponential tail, such as the fact that showers produced by nuclei or protons (or even photons) have a distribution of $X_{\max }$ that shows an exponential shape, dictated by the distribution of the primary interaction. The exponential model may be checked below the cut by comparing the actual number of events observed in the $[3 \sigma, 4 \sigma],[4 \sigma, 5 \sigma],[5 \sigma, 6 \sigma]$ and $[6 \sigma, 7 \sigma]$ regions, to the number of events predicted by extrapolating the fit done in the $[1 \sigma, 3 \sigma]$ region. The values are in good agreement as shown in Fig. 7, For our search sample (equivalent to two years of full detector data) we have an estimated background of 0.1 events for each multiplicity class that add up to a total of 0.3 events with a statistical uncer- 
Fisher distribution - low mult. $(4 \leq \mathbf{N} \leq 6)$

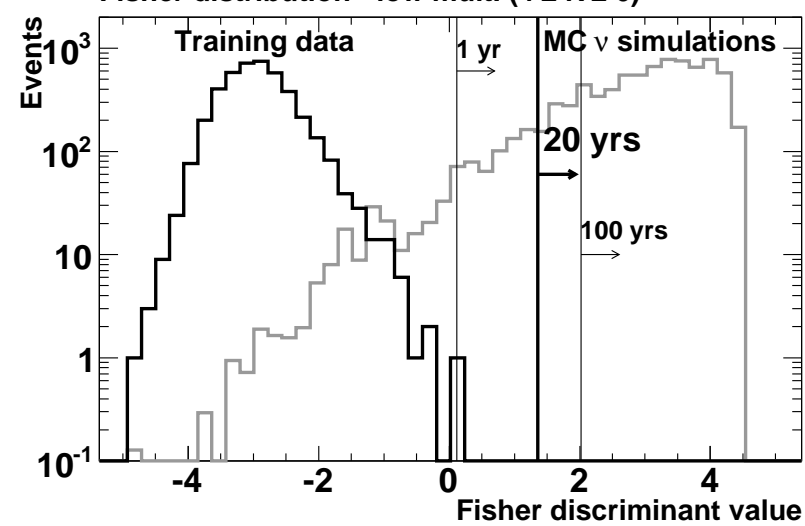

Fisher distribution - medium mult. $(\mathbf{7} \leq \mathbf{N} \leq \mathbf{1 1})$

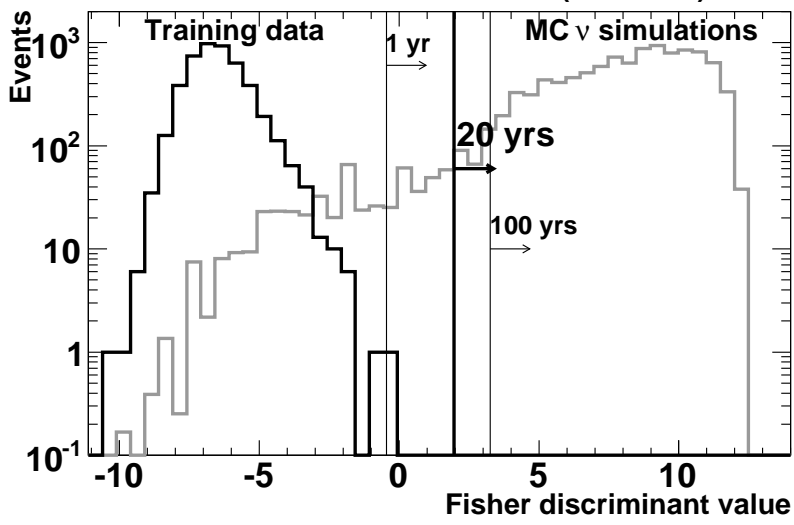

Fisher distribution - large mult. $(\mathbf{1 2} \leq \mathrm{N})$

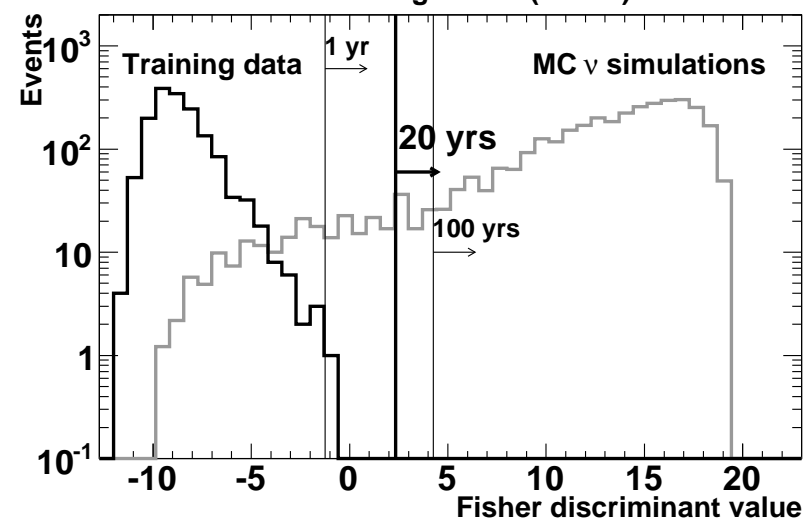

Fisher distribution - low mult. $(4 \leq \mathbf{N} \leq 6)$

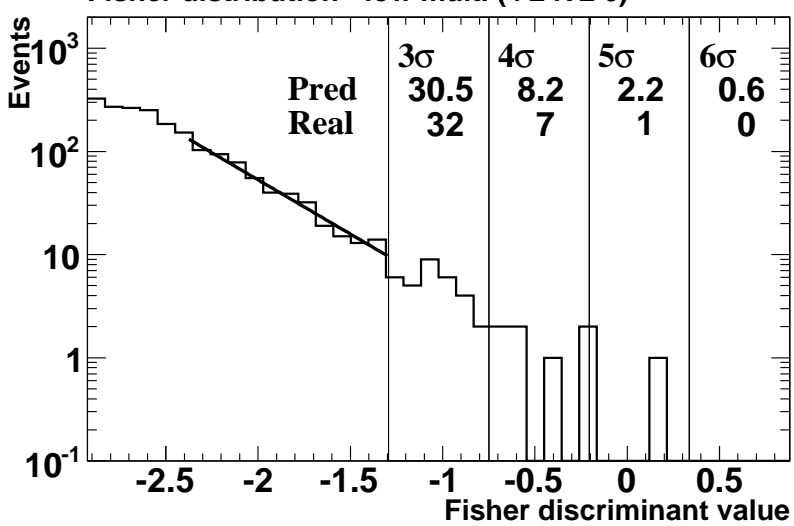

Fisher distribution - medium mult. $(\mathbf{7} \leq \mathbf{N} \leq \mathbf{1 1})$

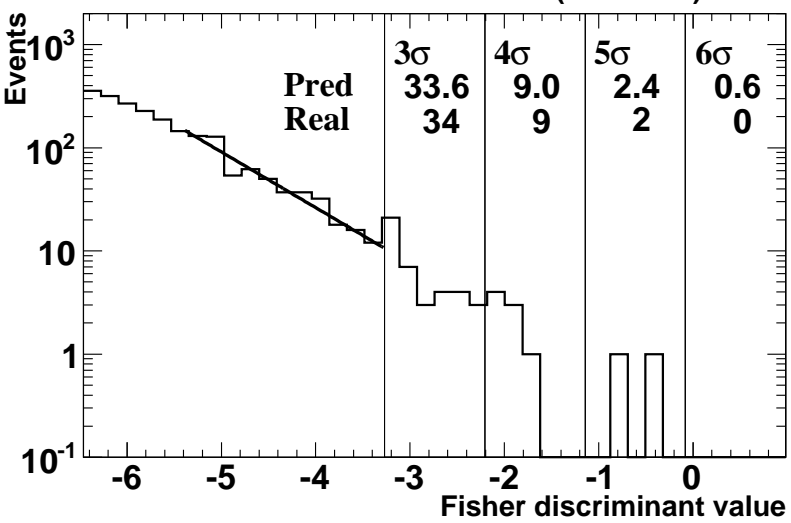

Fisher distribution - large mult. $(12 \leq \mathrm{N})$

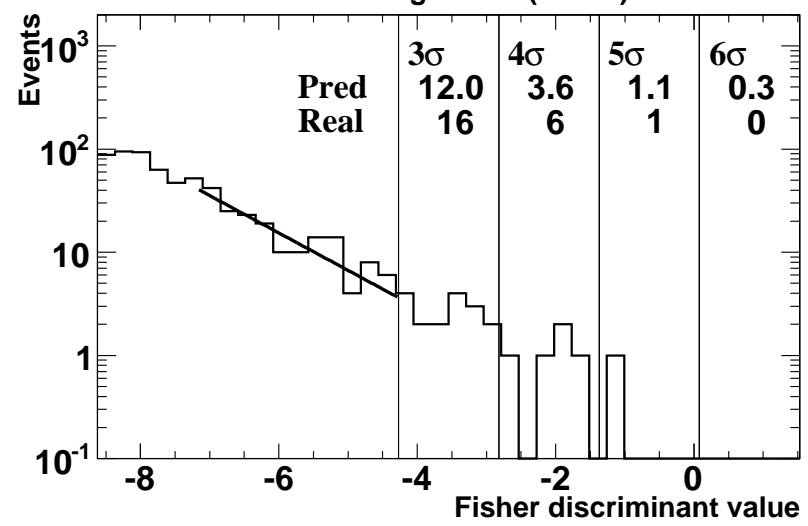

FIG. 7. Left panel: distribution of the Fisher discriminant (see text for details) for events with station multiplicity $4 \leq N \leq 6$ (top), $7 \leq N \leq 11$ (middle), $12 \leq N$ (bottom). Real data in the training period (1 Jan 04 - 31 Oct 07) describe the nucleonic background, while Monte Carlo simulated down-going neutrinos correspond to the signal. The vertical lines indicate the cut in the Fisher value that needs to be placed to have less than 1 event in each period of time (1 yr, $20 \mathrm{yr}, 100 \mathrm{yr})$. Right panel: fit of an exponential function to the distribution of the Fisher discriminant $\mathcal{F}$ for the training data over the $[1 \sigma, 3 \sigma]$ interval. The predicted (Pred.), see text, and actual (Real) number of events are given for each of the test zones $([3 \sigma, 4 \sigma]$, [4 $\sigma, 5 \sigma],[5 \sigma, 6 \sigma]$ and $[6 \sigma, 7 \sigma])$. 
tainty of $30 \%$. As we do not have at present a robust estimation of the background systematics we take a conservative approach and do not use this value to improve our flux upper limit.

As can be seen in Fig. 7 the identification cuts reject a small fraction of the neutrino events. Consequently, its choice has only a small impact on the neutrino identification efficiency. The neutrino-induced showers rejected by these cuts are those interacting far from the ground and similar to nucleonic-induced showers.

\section{IDENTIFICATION EFFICIENCIES AND EXPOSURE}

During the data taking, the array was growing and had sporadic local inefficiencies. Simulations of deep inclined neutrino showers indicate that besides an elongated pattern on the ground they have a large longitudinal uncertainty on the core position. For these reasons we cannot apply (as done in the case of vertical showers [25]) a geometrical method relying on the estimated position of the shower core within a triangle or hexagon of active stations at each time. Moreover, a shower can trigger the surface detector even if the core falls outside the array. Besides, for deep inclined showers the trigger and identification efficiencies depend not only on the shower energy and zenith angle but also on the depth of the first interaction. For these reasons a specific procedure was designed to compute the time dependent acceptance and the integrated exposure.

The instantaneous status of the array is obtained from the trigger counting files, which respond to the modifications of the array configuration at every second. To avoid having to cope with an enormous number of configurations, we approximate the calculation of the aperture by subdividing the search period in three-day intervals, and we select a reference array configuration to represent each. Once this is done we calculate the neutrino identification efficiencies and the aperture assuming that the array remains unchanged during each three-day interval. Each reference configuration was chosen so that this approximation, if wrong, underestimates the exposure by a small amount $(\sim 1 \%)$.

MC generated neutrino showers produced by AIRES were randomly distributed over an extended circular area around the array, such that a shower with a core falling outside this area has no chance to trigger the array. For each three-day configuration the FADC traces of the active Cherenkov stations were simulated, the local and global trigger conditions were applied and the events were processed through the same reconstruction and identification algorithms as the data (Sec. V).

Fig. 8 shows an example of a shower that would be a neutrino candidate in an ideal array, placed at four random positions on the circular surface defined above. Two of the realizations are effectively recognized as neutrino events in the real array for that particular layout. The

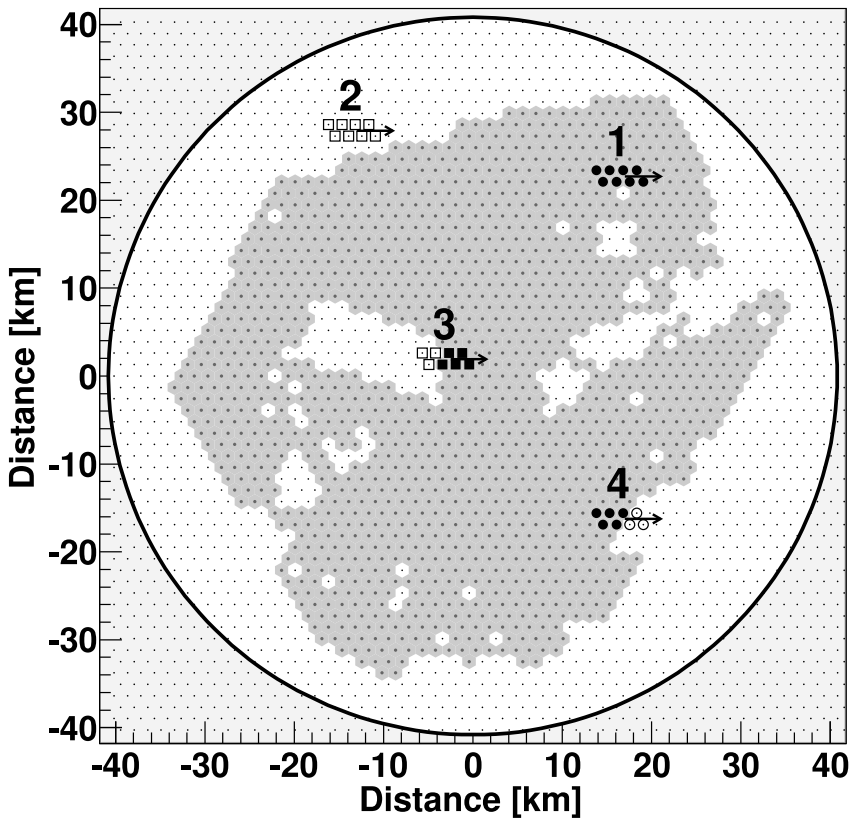

FIG. 8. An example of the result of placing the same deeply penetrating neutrino-induced shower at 4 different positions in an actual array configuration (shaded area) corresponding to 27 Oct 2007. The arrows indicate the azimuthal arrival direction of the shower, the dots represent the infinite ideal array and the circumference the extended area (see text). Solid symbols - either circles or squares - correspond to triggered stations of the simulated shower that are also on the actual array. Open symbols are stations that are not in the real array. Shower 1 is completely contained and identified as a neutrino. Shower 2 falls entirely outside the real array and it does not trigger the array. Although shower 3 triggers the array, it is not identified as a neutrino because the earliest three stations are not in the real array. Shower 4 loses some stations but keeps the earliest which are enough to identify the event as a neutrino.

other two are either not seen, or not identified as neutrinos.

Fig. 9 shows the efficiency (fraction of events which pass all steps) as a function of interaction depth in the atmosphere for neutrinos of $E_{\nu}=10^{18} \mathrm{eV}$ and $\theta=80^{\circ}$, in an "ideal" array without holes nor edges. There is essentially a plateau between a minimal depth (needed for the $\nu$-induced shower to reach a sufficient lateral expansion) and a maximal one (such that the electromagnetic component is almost extinguished at ground level) . Below and above this plateau, the efficiency drops rapidly to zero. In other words, for a given channel and given values of $\theta$ and $E_{\nu}$, there is a slice of atmosphere above the array where the interactions are detected and distinguished: the matter contained in this volume will be referred to as the "mass aperture" in the following.

For each three-day period, we compute the effective area defined as the integral of the efficiency over core 


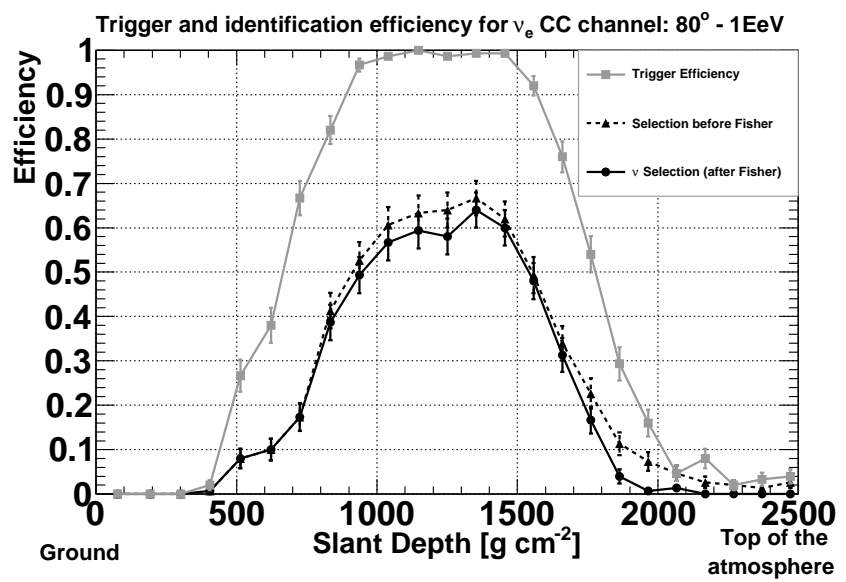

FIG. 9. Example of trigger and identification efficiency as a function of the slant depth of the interaction above the ground. Notice that the Fisher discriminant neutrino selection actually keeps most of the neutrino showers that pass the quality and reconstruction cuts discussed in Section IV.

position:

$$
A_{\text {eff }}\left(E_{\nu}, \theta, D, t\right)=\int \varepsilon\left(\vec{r}, E_{\nu}, \theta, D, t\right) \mathrm{d} A .
$$

The effective mass aperture $M_{\text {eff }}\left(E_{\nu}, t\right)$ is obtained by integrating over the injection depth $D$ and the solid angle:

$$
M_{\text {eff }}\left(E_{\nu}, t\right)=2 \pi \iint \sin \theta \cos \theta A_{\text {eff }}\left(E_{\nu}, \theta, D, t\right) \mathrm{d} \theta \mathrm{d} D .
$$

To compute this integral we perform a spline interpolation on the finite three-dimensional mesh where $A_{\text {eff }}$ is determined. The total mass aperture is then obtained summing $M_{\text {eff }}\left(E_{\nu}, t\right)$ over different configurations corresponding to a certain period of time. It is defined independently of the $\nu$-nucleon cross-section.

A combined exposure can be obtained by a summation over the search period:

$$
\mathcal{E}\left(E_{\nu}\right)=\sum_{i}\left[\omega^{i} \sigma^{i}\left(E_{\nu}\right) \int \frac{M_{\text {eff }}^{i}\left(E_{\nu}, t\right)}{m} \mathrm{~d} t\right]
$$

The sum runs over the three neutrino flavours (with fractions $\omega^{i}$ ) and the CC and NC interactions; $m$ is the mass of a nucleon. Here we assume a full $\nu_{\tau} \leftrightarrow \nu_{\mu}$ mixing, leading to $\omega^{i}=1$ for the three flavours.

We use the $\nu$-nucleon cross-section given in [37] (CSS hereafter) to compute the reference exposure of our search period. It is shown in Fig. 10 as a function of neutrino energy. In Table \ we also give the mass aperture integrated in time for all the considered channels, allowing the reader to compute the exposure using different cross-sections or flux models.

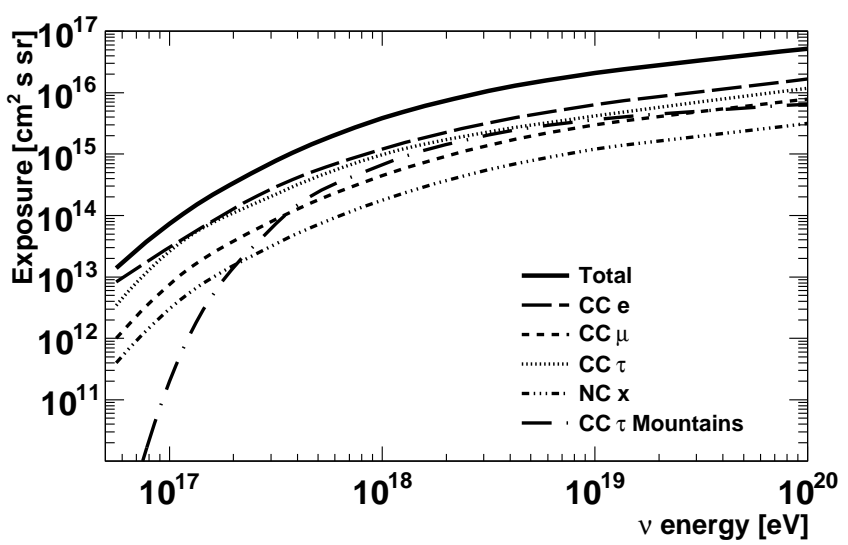

FIG. 10. SD Exposure for our search period for down-going neutrino-initiated showers. The total exposure is shown as a full line. The exposure for individual neutrino flavours and interaction channels is also shown.

\begin{tabular}{cccccc}
\hline \hline $\log E / \mathrm{eV}$ & $\nu_{e} \mathrm{CC}$ & $\nu_{\mu} \mathrm{CC}$ & $\nu_{\tau} \mathrm{CC}$ & $\nu_{x} \mathrm{NC}$ & $\nu_{\tau}$ Mount. \\
\hline 16.75 & $4.35 \cdot 10^{21}$ & $5.27 \cdot 10^{20}$ & $1.82 \cdot 10^{21}$ & $2.11 \cdot 10^{20}$ & - \\
17 & $1.27 \cdot 10^{22}$ & $3.16 \cdot 10^{21}$ & $1.09 \cdot 10^{22}$ & $1.26 \cdot 10^{21}$ & - \\
17.5 & $7.94 \cdot 10^{22}$ & $2.34 \cdot 10^{22}$ & $6.02 \cdot 10^{22}$ & $9.37 \cdot 10^{21}$ & $1.98 \cdot 10^{22}$ \\
18 & $2.17 \cdot 10^{23}$ & $8.01 \cdot 10^{22}$ & $1.77 \cdot 10^{23}$ & $3.20 \cdot 10^{22}$ & $1.21 \cdot 10^{23}$ \\
18.5 & $3.95 \cdot 10^{23}$ & $1.71 \cdot 10^{23}$ & $2.84 \cdot 10^{23}$ & $6.84 \cdot 10^{22}$ & $2.51 \cdot 10^{23}$ \\
19 & $5.44 \cdot 10^{23}$ & $2.56 \cdot 10^{23}$ & $3.58 \cdot 10^{23}$ & $1.03 \cdot 10^{23}$ & $3.13 \cdot 10^{23}$ \\
19.5 & $6.32 \cdot 10^{23}$ & $2.99 \cdot 10^{23}$ & $4.36 \cdot 10^{23}$ & $1.20 \cdot 10^{23}$ & $3.06 \cdot 10^{23}$ \\
20 & $7.29 \cdot 10^{23}$ & $3.45 \cdot 10^{23}$ & $5.19 \cdot 10^{23}$ & $1.38 \cdot 10^{23}$ & $2.82 \cdot 10^{23}$ \\
\hline \hline
\end{tabular}

TABLE I. Effective mass aperture integrated over time for the search period (1 Nov 2007 to 31 May 2010) for down-going neutrinos of the Pierre Auger Surface Detector (in units of [g sr s]).

\section{SYSTEMATIC UNCERTAINTIES}

The calculation of the mass aperture of the Auger Observatory for neutrino showers requires the input of several ingredients which we have selected from amongst conventionally used options. Some of these choices are directly related to the Monte Carlo simulation of the showers, i.e. generator of the first neutrino interaction, parton distribution function (PDF), air shower development and hadronic model. Others have to do with the precision of our knowledge of the topography of the mountains surrounding the Observatory, and some come from the limitations on the theoretical models estimating, for instance, the interaction cross-section or the $\tau$ energy loss at high energies. By adding linearly all these contributions, our estimate of the total systematic uncertainty on the exposure amounts to $+22 \%-46 \%$.

In the following subsections we discuss in detail the dependence of the exposure on each of the above mentioned choices by modifying the different ingredients one by one. 


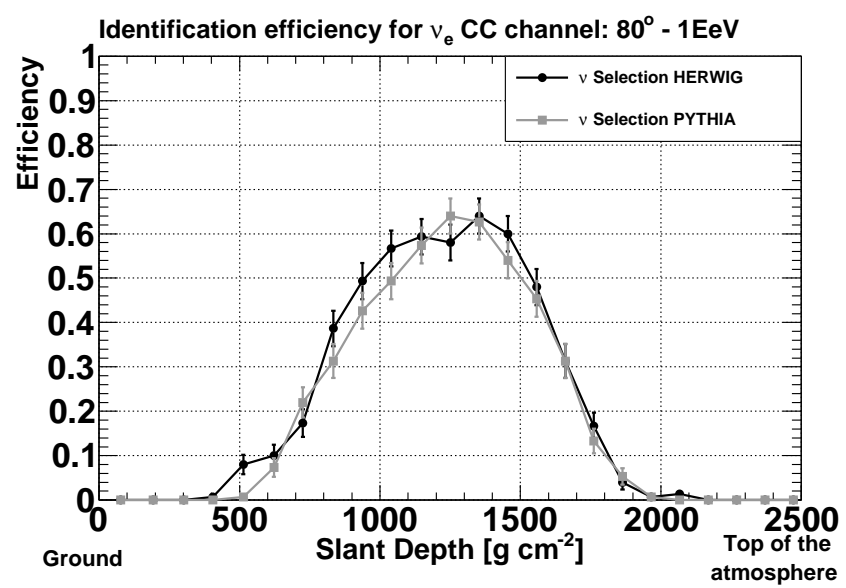

FIG. 11. Identification efficiency as function of the slant depth for systematic uncertainties studies. Comparison between interaction generators (HERWIG and PYTHIA). The rest of the Monte Carlo input parameters remain the same.

\section{A. Monte Carlo simulation of the shower}

The reference Monte Carlo neutrino sample was produced with HERWIG 6.5.10 [26] as interaction generator in combination with the CTEQ06m [38] parton distribution functions, AIRES 2.8 as shower simulator (thinning value of $10^{-6}$ ) and QGSJETII.03 [39] as hadronic model.

In order to assess the influence of this particular choice of models on the detector aperture, independent sets of $\mathrm{CC} \nu_{e}$ showers were generated at $1 \mathrm{EeV}$ and $80^{\circ}$ using different combinations of several interaction generators, PDFs, shower simulators, thinning values and hadronic models. We chose this particular energy and angle bin because it is the one that contributes the most to the limit.

In Fig. 11 we show, as an example, the detection efficiency as a function of the slant depth when using our reference options (HERWIG) and when changing only the interaction generator (PYTHIA). Since the shapes of the neutrino-selection efficiency curves remain similar, we can estimate the effect of changing the interaction generator by computing the integral of the curves and reporting the relative difference (RD) between them. The same procedure is applied to estimate the effect of changing other ingredients of the simulation. A summary of this RDs is given in Table II.

We observe that the changes in interaction generator, PDF, shower simulator and hadronic model brought about a decrease of the estimated aperture, with the choice of the shower simulation being the dominating effect. On the other hand, an improvement of the relative thinning level causes the opposite effect. Although we cannot recompute the aperture for all possible alternatives of the relevant ingredients, the relative differences reported in Table II serve as an estimate of the systematic dependence of our result on the simulation options.

\begin{tabular}{lllc}
\hline \hline Parameter & Reference & Modification & \multicolumn{1}{c}{ RD } \\
& $(\mathbf{A})$ & $(\mathbf{B})$ & $\frac{\int \mathbf{B}-\int \mathbf{A}}{\left(\int \mathbf{B}+\int \mathbf{A}\right) / \mathbf{2}}$ \\
\hline Interaction generator & HERWIG & PYTHIA [40] & $-7 \%$ \\
& & HERWIG++ [41] & $-7 \%$ \\
\hline PDF (gen. level) & CTEQ06m & MSTW [42] & $-7 \%$ \\
\hline Shower Simulator & AIRES & CORSIKA 6.9 [43] & $-17 \%$ \\
\hline Hadronic Model & QGSJETII & QGSJETI $[44]$ & $+2 \%$ \\
& & SIBYLL [45] & $-2 \%$ \\
& & SIBYLL $(E=0.3 \mathrm{EeV})$ & $-1 \%$ \\
& & SIBYLL $(E=3 \mathrm{EeV})$ & $-2 \%$ \\
& & SIBYLL $\left(\theta=85^{\circ}\right)$ & $0 \%$ \\
& & SIBYLL $\left(\theta=89^{\circ}\right)$ & $+4 \%$ \\
\hline \hline Thinning & & $10^{-7}$ & $+7 \%$ \\
\hline \hline
\end{tabular}

TABLE II. Summary of the relative differences (RD) between the reference calculation of the exposure and the calculations done changing one of the ingredients of the Monte Carlo simulations at a time. The RD were obtained for zenith angle $\theta=80^{\circ}$ and energy $E=1 \mathrm{EeV}$ unless otherwise stated. The statistical uncertainty of all the relative differences is $\pm 4 \%$.

For each category of potential systematic effects in Table II, we take the maximum observed RD as an estimate of the corresponding systematic uncertainty. A total systematic uncertainty of $+9 \%-33 \%$ on the exposure is obtained by linear addition of the maximum positive and negative deviations.

\section{B. $\quad \nu$-nucleon cross-sections and $\tau$ energy loss}

We adopted the uncertainty in the $\nu$-nucleon crosssection as calculated in [37. It translates into a $\pm 7 \%$ uncertainty in the total exposure. In any case, as mentioned above, Table I shows the Auger mass aperture for down-going neutrinos which does not depend on the $\nu$ cross-section; hence the expected neutrino event rate (and neutrino flux limit) can be computed as necessary for other models and values of the $\nu$ cross-section (see e.g. [46, 47]).

\section{Topography}

As explained in section ПII the actual topography surrounding the Observatory has been taken into account by detailed Monte Carlo simulations which include digital elevation maps. In principle, uncertainties due to different tau energy loss models should not be important for down-going neutrinos, but, due to the fact that the Pierre Auger Observatory is close to the Andes, a non negligible contribution to the event rate from down-going $\tau$ neutrinos interacting in the mountains and producing a $\tau$ lepton is expected (see Table III). The systematic error on the total reference exposure due to this channel amounts to $\pm 6 \%$, dominated by the uncertainties on the cross-section and energy loss models. 


\section{RESULTS AND DISCUSSION}

In this section we present the calculation of the upper limit to the diffuse flux of UHE $\nu$ s and compare our results to some selected model predictions and discuss the implications.

\section{A. Upper limit on the diffuse neutrino flux}

Once the multivariate algorithms and selection cuts defining a neutrino candidate were studied and tuned with the Monte Carlo simulations and the training data sample, we applied them to the search data sample. We first tested the compatibility between the shapes of the tails of the Fisher distributions during training and search periods by using an unbinned Kolmogorov hypothesis test, and found them to be in agreement with pvalues of $0.37,0.16$ and 0.17 for the small, medium and large multiplicity classes, respectively.

We found no candidate events in the search period (see Fig. 12). The highest test zone with events in the Fisher distribution of the search sample is the 6-7 sigma region. It has only one event and we expected 2.2 from the exponential fit to the test sample.

The expected number of events from a diffuse flux of neutrinos in a given energy range is given by:

$$
N_{\text {expected }}=\int_{E_{\min }}^{E_{\max }} \Phi\left(E_{\nu}\right) \mathcal{E}\left(E_{\nu}\right) \mathrm{d} E_{\nu},
$$

where $\mathcal{E}\left(E_{\nu}\right)$ is our reference exposure (eq. 3] and Fig. 10). The upper limit is derived for a differential neutrino flux $\Phi\left(E_{\nu}\right)=k \cdot E_{\nu}^{-2}$. Also, we assume that due to neutrino oscillations the diffuse flux is composed of electron, muon and $\tau$ neutrinos in the same proportion. We expect less than one background event after the neutrino selection procedure is applied to the data sample corresponding to the reference exposure (see section 5). Given the uncertainties of this estimate, the number of background events will be assumed to be zero, which results in a more conservative upper limit. A semi-Bayesian extension [48] of the Feldman- Cousins approach [49] is used to include the uncertainty in the exposure, giving an upper limit at $90 \%$ CL on the integrated flux of diffuse neutrinos of:

$$
k<1.74 \times 10^{-7} \mathrm{GeV} \mathrm{cm}^{-2} \mathrm{~s}^{-1} \mathrm{sr}^{-1} .
$$

The effect of including the systematics from $\mathrm{MC}, \nu-$ nucleon cross-sections and $\tau$ energy loss is to increase the limit by $15 \%$. The limit is quoted for a single neutrino flavour. The relative importance of each neutrino flavour in the determination of the upper limit can be derived from Table III, which gives the expected fractions of neutrinos in the selected sample according to their flavour and interaction channel. The largest contribution comes from $\nu_{e} \mathrm{CC}$. The second largest is $\nu_{\tau} \mathrm{CC}$, due to double-bang interactions and the large average fraction of energy going into the shower in the decay of the
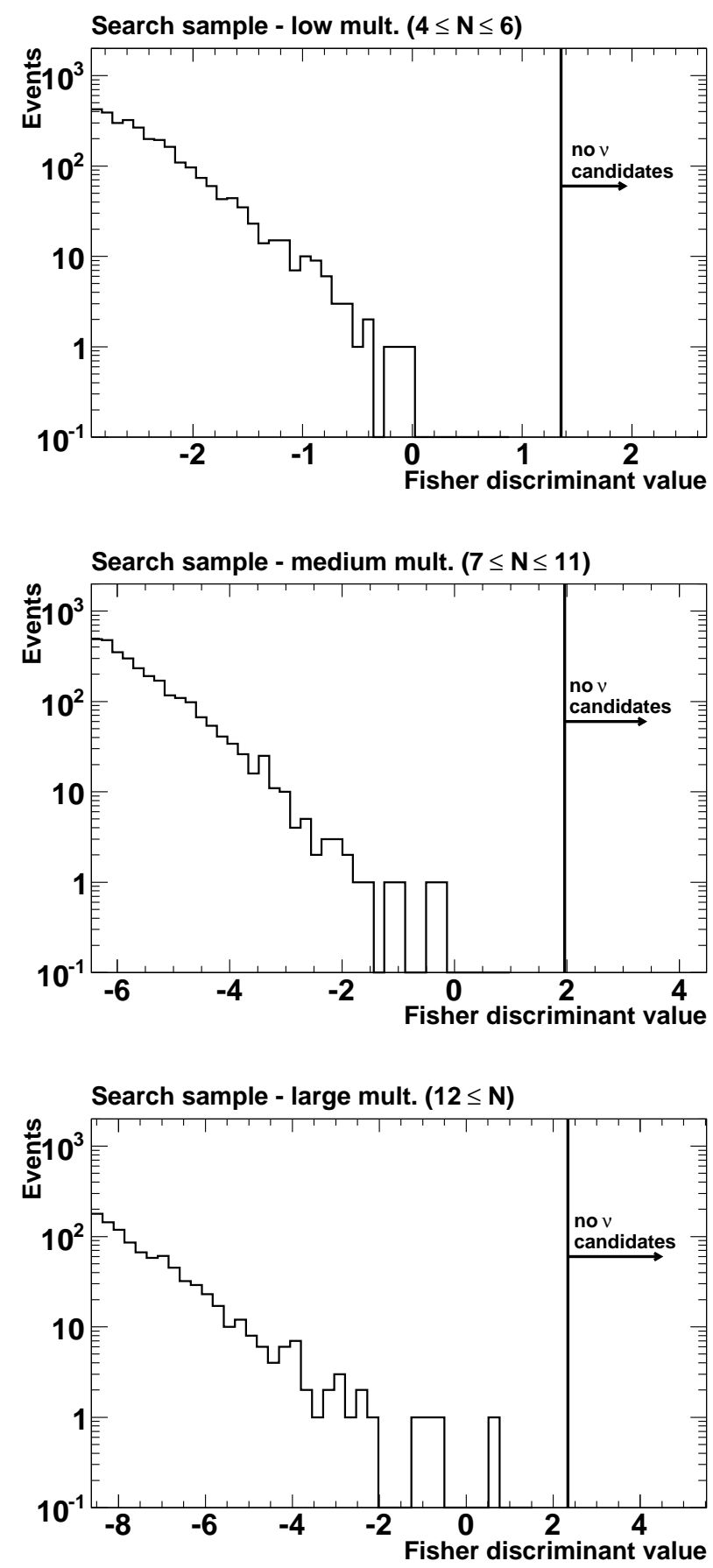

FIG. 12. Fisher distribution of the search sample (1 Nov 07 - 31 May 10, 2010) for events with multiplicity $4 \leq N \leq 6$ (top), $7 \leq N \leq 11$ (middle), $12 \leq N$ (bottom). No neutrino candidates are found.

$\tau$ lepton. Our result together with other experimental limits [50] is shown in Fig. 13.

Another usual way of presenting the upper bound is in the less-model-dependent differential form. It assumes that the diffuse neutrino flux behaves as $\sim 1 / E^{2}$ within energy bins of unity width on a natural logarithmic scale, 


\begin{tabular}{lccc}
\hline \hline Channel & CC & NC & Total \\
\hline$e$ & $33 \%$ & $5 \%$ & $38 \%$ \\
$\mu$ & $13 \%$ & $5 \%$ & $18 \%$ \\
$\tau$ air & $24 \%$ & $5 \%$ & $29 \%$ \\
$\tau$ mountains & $15 \%$ & & $15 \%$ \\
\hline Total & $85 \%$ & $15 \%$ & $100 \%$ \\
\hline \hline
\end{tabular}

TABLE III. Expected fractions of neutrinos in the selected sample according to their flavour and interaction channel (CC and NC). These fractions are derived assuming that electron, muon and $\tau$ neutrinos are in the same proportion in the diffuse flux.

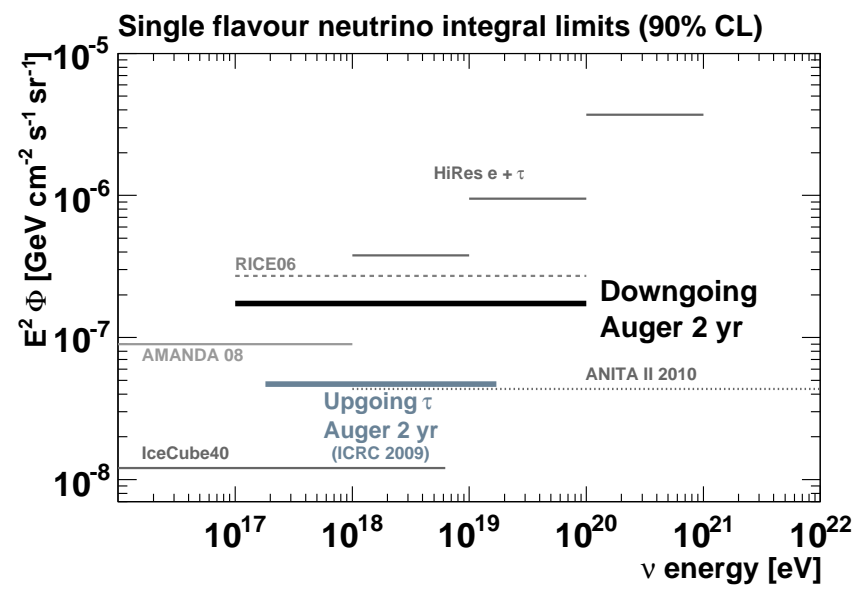

FIG. 13. Integrated upper limits (90\% CL) from the Pierre Auger Observatory for a diffuse flux of down-going $\nu$ in the period 1 Nov 2007 - 31 May 2010. For comparison up-going $\nu_{\tau}$ (1 Jan 2004 - 28 Feb 09) [13] and limits from other experiments [50] are also plotted.

and is given by $2.44 / \mathcal{E}\left(E_{\nu}\right) E_{\nu}$ accounting for statistical uncertainties only and assuming no background [51]. The differential limit obtained including systematic uncertainties is shown in Fig. 14 together with our previous result on up-going $\nu_{\tau}$ 13] and two theoretical predictions for cosmogenic neutrinos [6, 7]. We observe that we achieve maximum sensitivity in the $0.3-10 \mathrm{EeV}$ energy range.

\section{B. Model predictions}

There is a wide variety of models predicting fluxes of neutrinos with energies in the EeV range [1]. They are usually separated into three groups: cosmogenic neutrinos [e.g. 6, 7], neutrinos produced in accelerating sources [e.g. 53, 54] and neutrinos of exotic origin [e.g. 52]. In all these models there are parameters with unknown values which can change the spectral shape and strength of the flux. In Table IV we give the event rates

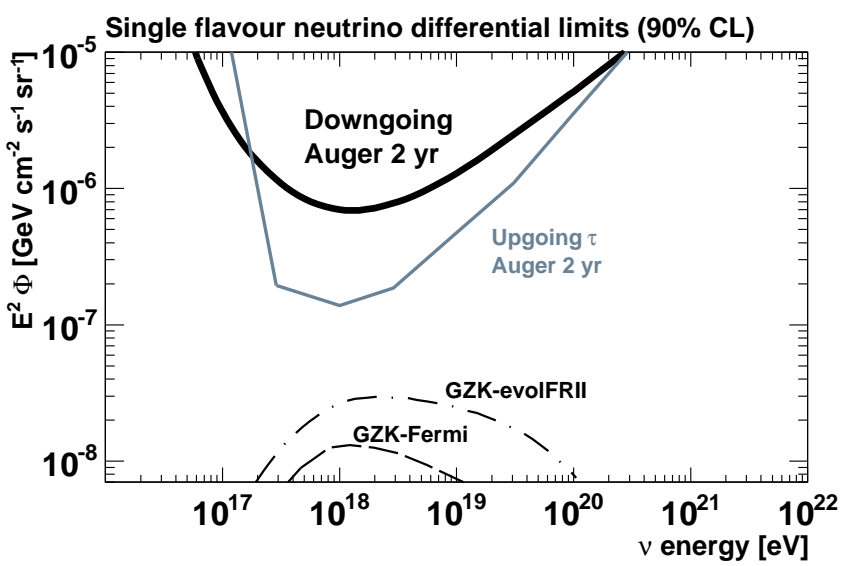

FIG. 14. Differential limits (90\% CL) from the Pierre Auger Observatory for a diffuse flux of down-going $\nu$ in the period 1 Nov 2007 - 31 May 2010 and up-going $\nu_{\tau}$ (1 Jan 2004 28 Feb 2009)[13]. For reference, two recent calculations of this flux are shown: "GZK-Fermi" [6] takes into account the Fermi-LAT constraint on the GZK cascade photons; the other "GZK-evolFRII" 7] adopts a strong source evolution model for FR-II galaxies, assumed to be the sources of UHECRs.

\begin{tabular}{lcc}
\hline \hline Reference & N expected & P. of obs. $\mathbf{~}$ \\
\hline GZK-Fermi & 0.1 & 0.9 \\
GZK-evolFRII & 0.3 & 0.7 \\
\hline MPR-max & 2.0 & 0.1 \\
BBR & 0.8 & 0.4 \\
\hline TD-Necklaces & 0.8 & 0.4 \\
Z-Burst & 7.8 & $4 \times 10^{-4}$ \\
\hline \hline
\end{tabular}

TABLE IV. Expected number of events using the current exposure of down-going $\nu$ measured by the Pierre Auger Observatory for several models $[6,67,52,54]$. The third column gives the probabilities of observing 0 events given that we expect $N$.

after folding these fluxes with our reference exposure.

Current theoretical flux predictions for cosmogenic neutrinos [6, 7] seem to be out of reach of our present sensitivity. Concerning neutrinos produced in accelerating sources there are popular models 53, 54] which predict event rates which could be detected in the next few years. Regarding exotic models [52], TD-Necklaces will be within our sensitivity range in one or two years, while Z-Burst models are already strongly disfavoured. Note that all such 'top down' models are also tightly constrained by the limits of the Pierre Auger Observatory on the photon fraction in UHECR [20]. 


\section{ACKNOWLEDGMENTS}

The successful installation, commissioning and operation of the Pierre Auger Observatory would not have been possible without the strong commitment and effort from the technical and administrative staff in Malargüe.

We are very grateful to the following agencies and organizations for financial support: Comisión Nacional de Energía Atómica, Fundación Antorchas, Gobierno De La Provincia de Mendoza, Municipalidad de Malargüe, NDM Holdings and Valle Las Leñas, in gratitude for their continuing cooperation over land access, Argentina; the Australian Research Council; Conselho Nacional de Desenvolvimento Científico e Tecnológico (CNPq), Financiadora de Estudos e Projetos (FINEP), Fundação de Amparo à Pesquisa do Estado de Rio de Janeiro (FAPERJ), Fundação de Amparo à Pesquisa do Estado de São Paulo (FAPESP), Ministério de Ciência e Tecnologia (MCT), Brazil; AVCR AV0Z10100502 and AV0Z10100522, GAAV KJB100100904, MSMT-CR LA08016, LC527, 1M06002, and MSM0021620859, Czech Republic; Centre de Calcul IN2P3/CNRS, Centre National de la Recherche Scientifique (CNRS), Conseil Régional Ile-de-France, Département Physique Nucléaire et Corpusculaire (PNC-IN2P3/CNRS), Département Sciences de l'Univers (SDU-INSU/CNRS), France; Bundesministerium für Bildung und Forschung (BMBF), Deutsche Forschungsgemeinschaft (DFG), Finanzmin- isterium Baden-Württemberg, Helmholtz-Gemeinschaft Deutscher Forschungszentren (HGF), Ministerium für Wissenschaft und Forschung, Nordrhein-Westfalen, Ministerium für Wissenschaft, Forschung und Kunst, BadenWürttemberg, Germany; Istituto Nazionale di Fisica Nucleare (INFN), Ministero dell'Istruzione, dell'Università e della Ricerca (MIUR), Italy; Consejo Nacional de Ciencia y Tecnología (CONACYT), Mexico; Ministerie van Onderwijs, Cultuur en Wetenschap, Nederlandse Organisatie voor Wetenschappelijk Onderzoek (NWO), Stichting voor Fundamenteel Onderzoek der Materie (FOM), Netherlands; Ministry of Science and Higher Education, Grant Nos. 1 P03 D 014 30, N202 090 31/0623, and PAP/218/2006, Poland; Fundação para a Ciência e a Tecnologia, Portugal; Ministry for Higher Education, Science, and Technology, Slovenian Research Agency, Slovenia; Comunidad de Madrid, Consejería de Educación de la Comunidad de Castilla La Mancha, FEDER funds, Ministerio de Ciencia e Innovación and Consolider-Ingenio 2010 (CPAN), Xunta de Galicia, Spain; Science and Technology Facilities Council, United Kingdom; Department of Energy, Contract Nos. DEAC02-07CH11359, DE-FR02-04ER41300, National Science Foundation, Grant No. 0450696, The Grainger Foundation USA; ALFA-EC / HELEN, European Union 6th Framework Program, Grant No. MEIF-CT-2005025057, European Union 7th Framework Program, Grant No. PIEF-GA-2008-220240, and UNESCO.
[1] F. Halzen and D. Hooper, Rep. Prog. Phys. 65, 1025 (2002); P. Bhattacharjee and G. Sigl, Phys. Rep. 327, 109 (2000); J. K. Becker, Phys. Rep. 458, 173 (2008).

[2] J. Abraham et al. [Pierre Auger Collaboration], Phys. Rev. Lett. 101, 061101 (2008); R. U. Abbasi et al. [HiRes], Phys. Rev. Lett. 100, 101101 (2008).

[3] V. Beresinsky and G. Zatsepin, Phys. Lett. B 28, 423 (1969).

[4] S. Yoshida and M. Teshima, Prog. Theor. Phys. 89, 833 (1993).

[5] R. Engel, D. Seckel, and T. Stanev, Phys. Rev. D 64, 093010 (2001).

[6] M. Ahlers, L. A. Anchordoqui, M. C. Gonzalez-Garcia, F. Halzen and. S. Sarkar, Astropart. Phys. 34, 106 (2010).

[7] K. Kotera, D. Allard and A. V. Olinto, JCAP 10, 013 (2010).

[8] V. Berezinsky, A. Gazizov, M. Kachelriess and S. Ostapchenko, Phys. Lett. B 695, 13 (2011).

[9] A. Achterberg et al. [IceCube Collaboration], Astropart. Phys. 26, 155 (2006).

[10] J. A. Aguilar et al. [ANTARES Collaboration], Phys. Lett. B 696 16, (2011).

[11] P. W. Gorham et al. [ANITA Collaboration], Phys. Rev. Lett. 103, 051103 (2009); Astropart. Phys. 32, 10 (2009).

[12] J. Abraham et al. [Pierre Auger Collaboration], Phys. Rev. Lett. 100, 211101 (2008).

[13] J. Tiffenberg [Pierre Auger Collaboration], in Proceedings of the 31st International Cosmic Ray Conference, Lodz
(2009), \#0180, arXiv:0906.2347.

[14] D. Gora [Pierre Auger Collaboration], in Proceedings of the 31st International Cosmic Ray Conference, Lodz (2009), \#0077, arXiv:0906.2319.

[15] X. Bertou, P. Billoir, O. Deligny, C. Lachaud, and A. Letessier-Selvon, Astropart. Phys 17, 183 (2002).

[16] J. Abraham et al. [Pierre Auger Collaboration], Phys. Rev. D 79, 102001 (2009).

[17] J. Alvarez-Muñiz [Pierre Auger Collaboration], in Proceedings of the 30th International Cosmic Ray Conference, Mérida Vol. 4, (2007), p. 607; I. Valiño (PhD thesis) Univ. de Santiago de Compostela, ISBN: 9788497509664 , (2008).

[18] K. S. Capelle, J. W. Cronin, G. Parente and E. Zas, Astropart. Phys. 8, 321 (1998); P. Billoir [Pierre Auger Collaboration], J. Phys. Conf. Ser. 203, 012125 (2010).

[19] J. Abraham et al. [Pierre Auger Collaboration], Phys. Rev. Lett. 104, 091101 (2010).

[20] J. Abraham et al. [Pierre Auger Collaboration], Astropart. Phys. 27, 155 (2007); J. Abraham et al. [Pierre Auger Collaboration], Astropart. Phys. 29, 243 (2008); J. Abraham et al. [Pierre Auger Collaboration], Astropart. Phys. 31, 399 (2009).

[21] J. Abraham et al. [Pierre Auger Collaboration] Nucl. Instr. and Meth. A 523, 50 (2004).

[22] I. Allekotte et al. [Pierre Auger Collaboration], Nucl. Instr. and Meth. A 586, 409 (2008).

[23] J. Abraham et al. [Pierre Auger Collaboration], Nucl. Instr. and Meth. A 620, 227 (2010). 
[24] X. Bertou et al. [Pierre Auger Collaboration], Nucl. Instr. and Meth. A 568, 839 (2006).

[25] J. Abraham et al. [Pierre Auger Collaboration], Nucl. Instr. and Meth. A 613, 29 (2010).

[26] G. Corcella, I. G. Knowles, G. Marchesini, S. Moretti, K. Odagiri, P. Richardson, M. H. Seymour and B. R. Webber, HERWIG 6.5, JHEP 0101 (2001).

[27] R. Decker, S. Jadach, J. H. Kuhn and Z. Was, Comput. Phys. Commun. 76, 361 (1993).

[28] S. Sciutto, AIRES. Available from http://www.fisica unlp.edu.ar/auger/aires/.

[29] S. Argirò et al. [The Offline group - Pierre Auger Collaboration] Nucl. Instr. and Meth. A, 580, 1485 (2007).

[30] T. G. Farr et al., Rev. Geophys. 45, 33 (2007).

[31] O. Blanch Bigas, O. Deligny, K. Payet, and V. Van Elewyck, Phys. Rev. D 77, 103004 (2008).

[32] A. M. Hillas, in Proceedings of the 17th International Cosmic Ray Conference, Paris Vol. 8, (1981) p. 193.

[33] P. Billoir, Astropart. Phys. 30, 270 (2008).

[34] S. Agostinelli et al., Nucl. Instr. and Meth. A 506, 250 (2003); J. Allison et al., IEEE Trans. Nucl. Sci. 53, 270 (2006). See also http://geant4.web. cern.ch/geant4/.

[35] R. Fisher, Ann. of Eugenics 7, 179 (1936).

[36] B. Roe, PHYSTAT-2003-WEJT003, 215 (2003).

[37] A. Cooper-Sarkar and S. Sarkar, JHEP 0801, 075 (2008).

[38] J. Pumplin, D. R. Stump, J. Huston, H. L. Lai, P. Nadolsky and W. K. Tung, JHEP 0207, 012 (2002).

[39] S. Ostapchenko, Nucl.Phys. B - Proc. Supp. 151, 143 (2006); S. Ostapchenko, Nucl.Phys. B - Proc. Supp. 151, 147 (2006).

[40] T. Sjöstrand, S. Mrenna and P. Skands, Comput. Phys. Commun. 178, 11, 852 (2008).

[41] M. Bähr et al., Eur. Phys. J. C 58, 639 (2008).
[42] A. D. Martin, W. J. Stirling, R. S. Thorne and G. Watt, Eur. Phys. J. C 63, 189 (2009).

[43] D. Heck, J. Knapp, J. N. Capdevielle, G. Schatz and T. Thouw, Report FZKA 6019 (1998).

[44] N. Kalmykov and S. Ostapchenko, Phys. Atom. Nucl. 56, 346 (1993).

[45] R. Engel, T. K. Gaisser, T. Stanev and P. Lipari, in Proceedings of the 26th International Cosmic Ray Conference, Salt Lake City Vol. 1 (1999) p. 415.

[46] A. Connolly, R. S. Thorne and D. Waters, Phys. Rev. D 83, 113009 (2011).

[47] A. Cooper-Sarkar, P. Mertsch and S. Sarkar, arXiv:1106.3723, (2011).

[48] J. Conrad, O. Botner, A. Hallgren and C. Perez de los Heros, Phys. Rev. D 67 (2003) 12002.

[49] G. J. Feldman and R. D. Cousins, Phys. Rev. D 57, 3873 (1998).

[50] M. Ackermann et al. [AMANDA], Astrophys. J. 675, 1014 (2008); R. Abbasi et al. [IceCube], Phys. Rev. D 83,092003 (2011); I. Kravchenko et al. [RICE], Phys. Rev. D 73, 082002 (2006); P. W. Gorham et al. [ANITAII Collaboration], Phys. Rev. D 82022004 (2010), Erratum arXiv:1011.5004 1 [astro-ph]; R. Abbasi et al. [HiRes], Astrophys. J. 684, 790 (2008); K. Martens [HiRes], arXiv:0707.4417.

[51] L. A. Anchordoqui, J. L. Feng, H. Goldberg and A. D. Shapere, Phys. Rev. D 66, 103002 (2002).

[52] O. E. Kalashev, V. A. Kuzmin, D. V. Semikoz and G. Sigl, Phys. Rev. D 66, 063004 (2002).

[53] K. Mannheim, R. J. Protheroe and J. P. Rachen, Phys. Rev. D 63 23003, (2000).

[54] J. K. Becker, P. L. Biermann and W. Rhode, Astropart. Phys. 23 355, (2005). 